OPEN ACCESS

Edited by:

Alejandro Cabezas-Cruz,

Institut National de la Recherche

Agronomique (INRA), France

Reviewed by:

Sara Moutailler

Agence Nationale de Sécurité

Sanitaire de l'Alimentation,

de l'Environnement et du Travail

(ANSES), France

Jan Kopecky

University of South Bohemia, Czechia

Jennifer Patricia Richardson,

Institut National de la Recherche

Agronomique (INRA), France

*Correspondence:

Saravanan Thangaman

Thangams@upstate.edu

Specialty section:

This article was submitted to

Virus and Host

a section of the journal

Frontiers in Cellular and Infection

Microbiology

Received: 25 October 2019

Accepted: 21 January 2020

Published: 18 February 2020

Citation:

Hart CE, Ribeiro JM, Kazimirova M and Thangamani S (2020) Tick-Borne Encephalitis Virus Infection Alters the Sialome of Ixodes ricinus Ticks During the Earliest Stages of Feeding.

Front. Cell. Infect. Microbiol. 10:41.

doi: 10.3389/fcimb.2020.00041

\section{Tick-Borne Encephalitis Virus} Infection Alters the Sialome of Ixodes ricinus Ticks During the Earliest Stages of Feeding

\author{
Charles E. Hart ${ }^{1,2,3}$, Jose M. Ribeiro ${ }^{4}$, Maria Kazimirova ${ }^{5}$ and Saravanan Thangamani ${ }^{1,2,6 *}$ \\ ${ }^{1}$ SUNY Center for Environmental Health and Medicine, SUNY Upstate Medical University, Syracuse, NY, United States, \\ ${ }^{2}$ Institute for Global Health and Translational Science, SUNY Upstate Medical University, Syracuse, NY, United States, ${ }^{3}$ The \\ Institute for Translational Science, University of Texas Medical Branch, Galveston, TX, United States, ${ }^{4}$ Laboratory of Malaria \\ and Vector Research, National Institute of Allergy and Infectious Diseases, Bethesda, MD, United States, ${ }^{5}$ Institute of \\ Zoology, Slovak Academy of Sciences, Bratislava, Slovakia, ${ }^{6}$ Department of Microbiology and Immunology, SUNY Upstate \\ Medical University, Syracuse, NY, United States
}

Ticks are hematophagous arthropods that transmit a number of pathogens while feeding. Among these is tick-borne encephalitis virus (TBEV), a flavivirus transmitted by Ixodes ricinus ticks in the temperate zone of Europe. The infection results in febrile illness progressing to encephalitis and meningitis with a possibility of fatality or long-term neurological sequelae. The composition of tick saliva plays an essential role in the initial virus transmission during tick feeding. Ticks secrete a diverse range of salivary proteins to modulate the host response, such as lipocalins to control the itch and inflammatory response, and both proteases and protease inhibitors to prevent blood coagulation. Here, the effect of viral infection of adult females of Ixodes ricinus was studied with the goal of determining how the virus alters the tick sialome to modulate host tissue response at the site of infection. Uninfected ticks or those infected with TBEV were fed on mice and removed and dissected one- and 3-h post-attachment. RNA from the salivary glands of these ticks, as well as from unfed ticks, was extracted and subjected to next-generation sequencing to determine the expression of key secreted proteins at each timepoint. Genes showing statistically significant up- or down-regulation between infected and control ticks were selected and compared to published literature to ascertain their function. From this, the effect of tick viral infection on the modulation of the tick-host interface was determined. Infected ticks were found to differentially express a number of uncategorized genes, proteases, Kunitz-type serine protease inhibitors, cytotoxins, and lipocalins at different timepoints. These virus-induced changes to the tick sialome may play a significant role in facilitating virus transmission during the early stages of tick feeding.

Keywords: vector-skin interface, tick-borne encephalitis virus, tick sialome, tick feeding, immunomodulation 


\section{INTRODUCTION}

Ticks are obligate hematophagous arthropods of the order Ixodida that transmit a variety of disease agents to humans and animals. In Europe, Russia, and Northern Asia, tick-borne viral diseases are a major public health concern. Of particular concern is tick-borne encephalitis virus (TBEV), a flavivirus endemic to Europe and northern Asia which is vectored by Ixodes ricinus in Europe and Ixodes persulcatus in parts of Eastern Europe and Asiatic Russia. Upon TBEV-infected tick feeding, the virus initially replicates in host skin fibroblasts (Hermance et al., 2016) and macrophages (Labuda et al., 1996). The virus is then transported by these macrophages to the draining lymph nodes, where it replicates before entering the blood and progressing to the remainder of the body. This results in febrile illness that may progress to neuroinvasive disease with meningitis or in more severe cases to encephalitis or myeloencephalitis (Zavadska et al., 2018). Tick-borne encephalitis (TBE) is diagnosed in about 12,000 people per year (World Health Organization, 2011), although the rate of exposure is thought to be substantially higher. The fatality rate of the European subtype of TBE (transmitted by I. ricinus) is $1-3 \%$, with over $40 \%$ of neuroinvasive cases resulting in long-term neurological sequelae.

Ticks are pool-feeders. To facilitate the feeding process, ticks secrete to the feeding pool a complex mixture of compounds in their saliva, containing hundreds of proteinaceous, and non-proteinaceous molecules that modulate host hemostasis, inflammation, immune reactions, and wound healing. The expression of the tick salivary factors undergoes significant and dynamic temporal regulation, where different factors are expressed and secreted throughout the feeding process (Francischetti et al., 2009; Šimo et al., 2017). These complex processes allow the ticks to remain attached for a prolonged period and compensate for different host defense mechanisms and requirements of the tick feeding cycle. Tick salivary molecules can be grouped into major categories based on their function, such as lipocalins (acting as kratagonists of biogenic amines and prostanoids), metalloproteases (having fibrinolytic activity), Kunitz-domain containing peptides (having putative sodium channel blocking or anticlotting activities), basic tail polypeptides (a unique protein family having anti-thrombin or plasminogen-activating activities), the salp-15 family (having immunosuppressive activity), the Isac family (having anticomplement activity), glycine-rich peptides (immunogenic components of the tick cement) (Francischetti et al., 2009; Blisnick et al., 2017; Chmelar et al., 2017; Šimo et al., 2017). Interestingly, not all molecules are secreted simultaneously (Kotsyfakis et al., 2015). This indicates that ticks adapt the composition of their sialomes (from the Greek, sialo = saliva) to the time of feeding, to their hosts, to the presence of pathogens, or to stress signals (Liu et al., 2014; Chmelar et al., 2016; Šimo et al., 2017; Tirloni et al., 2019). However, the mechanism of this adaptation is unknown.

In addition to creating a favorable microenvironment to acquire blood, the immunomodulatory functions of tick salivary molecules have been demonstrated to facilitate pathogen transmission. Tick saliva initially alters the inflammatory state of the inoculation site by altering the expression of cytokines (Thangamani et al., 2017) to increase neutrophil and monocyte (Hermance et al., 2016) influx. This results in an inflammatory environment that is conducive to viral invasion and macrophagedriven replication and dissemination (Labuda et al., 1996; Hermance et al., 2016). Elements of the tick saliva can also alter the behavior of dendritic cells to increase the replication of TBEV, in turn accelerating viral dissemination through the lymphatic system (Fialova et al., 2010; Lieskovska et al., 2018). Part of this effect appears to be due to lipocalins which target dendritic cells by binding to surface cholesterol (Roversi et al., 2017). Other affected cells include leukocytes (Rodriguez-Valle et al., 2013), natural killer cells, and other skin residents. Additional salivary factors can alter inflammation by targeting pro-inflammatory signaling molecules such as histamine, serotonin, leukotrienes, and complement (Francischetti et al., 2009; Šimo et al., 2017; Štibrániová et al., 2019).

The presence of tick saliva has been shown to increase both the acquisition and transmission of viruses. For example, ticks fed on animals inoculated with Thogoto virus have been shown to acquire infection at a higher rate when the inoculate also contained tick salivary gland extract (SGE) (Jones et al., 1989); the same has been observed with TBEV (Labuda et al., 1993). Experiments with Powassan virus (POWV), which is closely related to TBEV, had demonstrated that the amount of virus required to produce lethal animal infection was significantly lower when tick SGE was present (Hermance and Thangamani, 2015). This suggests that tick-borne viruses have evolved to exploit immunological events in the vertebrate hosts evoked by ticks to facilitate their feeding (Kazimírová et al., 2017). It is also plausible that the infection and replication of viruses in tick salivary glands induce alterations that enhance the transmission properties of tick saliva. For example, in contrast to uninfected ticks, the presence of POWV has been shown to increase skin inflammation during tick feeding (Hermance et al., 2016), which in turn increased the influx of macrophages and may potentially accelerate early viral infection and dissemination through the lymphatic system.

The potential of tick-borne viruses to alter the immunomodulatory properties of tick saliva to further enhance their own pathogenicity has not previously been accounted for in experiments involving the effect of tick saliva on viral transmission. This information is of great importance to understand the processes at the tick-host-virus interface and how these factors interact to produce early infection and systemic disease. In this manuscript, we describe for the first time the dynamic expression of tick salivary factors during the early stages of TBEV transmission and the implications of the components of the saliva of infected ticks in creating an immunomodulated feeding site.

\section{MATERIALS AND METHODS}

\section{Ticks and Animals}

Ixodes ricinus ticks were obtained from a laboratory colony maintained at the Institute of Zoology, Slovak Academy of 
Sciences (Bratislava, Slovakia). BALB/c mice (females, 5-weeksold) were purchased from Dobrá Voda Breeding Station (Institute of Experimental Pharmacology and Toxicology, Slovak Academy of Sciences). The mice were housed at the Institute of Virology (BMC SAS) under standard conditions. Food and water were provided ad libitum. Mice were 6 weeks old at the start of the experiments, and at the end of the experiments they were euthanized by cervical dislocation under anesthesia induced by carbon dioxide.

\section{Ethics Statement}

The experiments involving laboratory mice were performed in accordance with the animal use protocol approved by the State Veterinary and Food Administration of the Slovak Republic (permit number 1335/12-221) and the Institute of Virology, Biomedical Research Center of the Slovak Academy of Sciences (BMC SAS).

\section{Tick Infection With TBEV, Feeding, and Dissection}

F1 generation of laboratory-bred I. ricinus females were used for virus inoculation. TBEV (Hypr strain prepared as a $10 \%$ mouse brain suspension of $1.1 \times 10^{9} \mathrm{PFU} / \mathrm{ml}$ in Leibovitz's L15 medium) was provided by the Institute of Virology BMC SAS. Unfed I. ricinus females were inoculated with TBEV $(5.5 \times$ $10^{4} \mathrm{PFU}$ per tick) through the coxal plate of the second pair of legs by a digital microinjector TM system (MINJ-D-CE; Tritech Research, Inc., USA) (for details see Thangamani et al., 2017) and subsequently kept at room temperature and $85 \%$ relative humidity in a desiccator for 21 days. The infection rate achieved by this procedure is $\sim 100 \%$ (Slovák et al., 2014).

Two groups of BALB/c mice ( $n=6$ each) were infested with TBEV-infected or uninfected (control) I. ricinus females, respectively. The ticks were placed in small neoprene capsules glued on the shaved backs of the mice (two capsules per mouse, four tick females per capsule) (Thangamani et al., 2017). Ticks in each capsule were allowed to feed for either 1 or $3 \mathrm{~h}$. After the allotted feeding time, mice ( $n=3$ per group and timepoint) were euthanized, and the attached ticks from one of the capsules were removed from the skin. The ticks and skin from the other capsules were used for the study by Thangamani et al. (2017). Ticks were dissected in chilled sterile PBS, pH 7.2, and their salivary glands were stored individually in either RNALater (uninfected ticks) or Tri Reagent (TBEV-infected ticks). Along with feeding ticks, salivary glands of unfed ticks (both TBEVinfected and uninfected) were prepared as described above. Samples in RNALater and TriReagent were stored at $-80^{\circ} \mathrm{C}$.

\section{Next Generation Sequencing}

Three ticks (one tick per mouse) for each treatment condition (infected or non-infected) and each of the feeding timepoints were used in this study. RNA from the salivary glands of these ticks were extracted as described by us earlier (Hermance and Thangamani, 2015), and pooled prior to Illumina Next Generation Sequencing analysis. Illumina TruSeq v2 sample preparation kits were used for the RNA-Seq library construction. Each sample library was uniquely indexed to allow combining libraries during sequencing, and subsequent separation postsequencing. Next generation sequencing (NGS) was performed at the UTMB NGS core facility. Sample libraries were analyzed by the Illumina HiSeq 1,500 using a $2 \times 50$ base paired end run protocol, with TruSeq v3 sequencing-by-synthesis chemistry.

\section{Bioinformatic Analysis}

Bioinformatic analyses were conducted following the methods described previously (Chagas et al., 2013; Ribeiro et al., 2014), with some modifications. Briefly, the fastq files were trimmed of low-quality reads $(<20)$, removed from contaminating primer sequences and concatenated for single-ended assembly using the Abyss (using k parameters from 25 to 95 in 10 fold increments) (Birol et al., 2009) and Trinity (Grabherr et al., 2011) assemblers. The combined fasta files were further assembled together with previous assemblies from I. ricinus (Schwarz et al., 2013; Kotsyfakis et al., 2015) using an iterative blast and CAP3 pipeline as previously described Karim et al. (2011). Coding sequences (CDS) were extracted based on the existence of a signal peptide in the longer open reading frame (ORF) and by similarities to other proteins found in the Refseq invertebrate database from the National Center for Biotechnology Information (NCBI), proteins from Acari deposited at NCBI's GenBank and from SwissProt. Reads for each library were mapped on the deducted CDS using the program blastn with a word size of 25 , minimum identity of $97 \%$ and only one gap allowed. Functional classification of the transcripts was achieved by scanning the output of the different blast and rpsblast results using a vocabulary of $\sim 400$ words, the e value of the result and a result coverage $>75 \%$. The classification of "unknown" was given if no informative match could be found. Read counts for each CDS were transformed to FPKM values. FPKM is a measure proportional to the relative molar abundance of each transcript.

Pairwise statistical comparisons between infected and uninfected libraries at the three different physiological states were assessed by a chi-squared test. The Bonferroni and FDR correction (Benjamini and Hochberg, 1995) were applied using the $P$-value package version 3.3.0 from the $\mathrm{R}$ software package (Team R. C., 2013). The normalized reads rate was determined by the expressions $\mathrm{r} 1 \times \mathrm{R} 2 /[\mathrm{R} 1 \times(\mathrm{r} 2+1)]$ e $\mathrm{r} 2 \times \mathrm{R} 1 /[\mathrm{R} 2 \times$ $(\mathrm{r} 1+1)]$, in which $\mathrm{r} 1$ and $\mathrm{r} 2$ are the reads for each library $(\mathrm{T}-$ and $\mathrm{T}+$ ) mapping to a particular transcript and $\mathrm{R} 1$ and R2 the number of total reads from each library mapped over all the CDS. One unit was added to the denominator to avoid division by zero. The Kruskall-Wallis statistical test (within the $\mathrm{R}$ package) was used to test for significant differences in FPKM values between transcripts within the different functional classes.

\section{Class Organization and Categorization}

The list of identified transcripts was narrowed based on several parameters. Firstly, contigs with RPKM values of $<10$ were excluded. Transcripts were then selected based on having a p-value smaller than 0.05 for at least one timepoint (unfed ticks, $1 \mathrm{~h}$ post-attachment, and $3 \mathrm{~h}$ post attachment). The list was further narrowed to only proteins that were likely to be secreted as defined by the presence of secretion-related sequences or the 


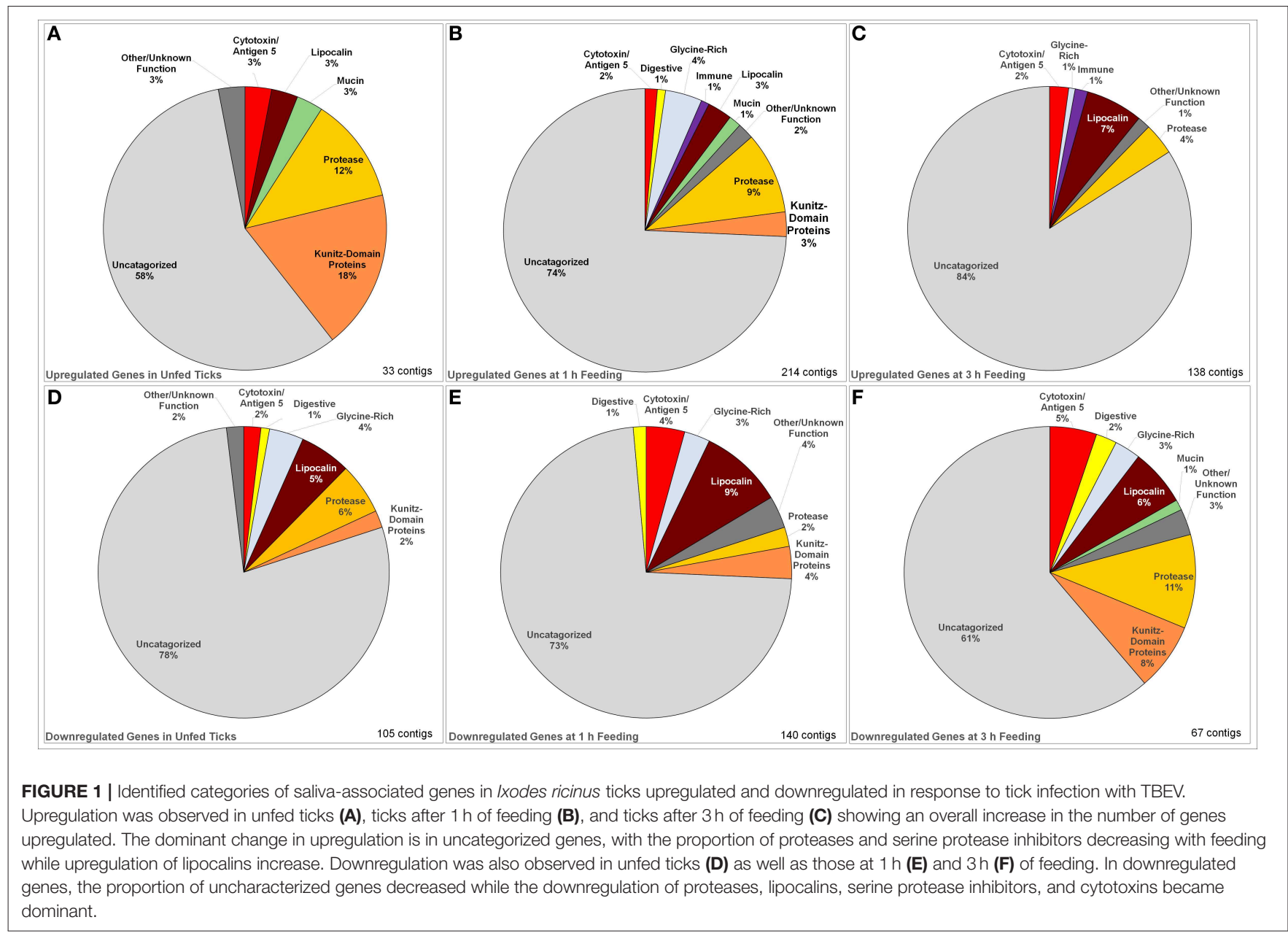

predicted function of the protein being identified as salivary in nature (i.e., lipocalins or cystatins).

These genes were then organized based on functional class, the results of which are presented in Figure 1, with a specific focus only on those components that are transferred to the host via saliva to modulate the host response to tick feeding (as opposed to metabolic or signaling genes within the salivary gland itself). Genes were identified as upregulated if expression in infected ticks was at least 10-fold higher than in uninfected ticks for at least one timepoint. Downregulated genes were defined as any that were downregulated below 0.1 -fold during active tick feeding at 1 and 3 -h timepoints as well as in cases where a gene was downregulated at any timepoint and upregulated at another. This relatively high 10 -fold threshold was used to avoid false positives. This list was compiled into Tables 1, 2.

Comparisons were derived from comparing expression changes of each functional class of predicted secreted salivary proteins at each timepoint.

\section{Evolutionary Relationships of Ixodes ricinus Lipocalins}

The evolutionary history was inferred using the NeighborJoining method (Saitou and Nei, 1987). The bootstrap consensus tree inferred from 1000 replicates is taken to represent the evolutionary history of the taxa analyzed (Felsenstein, 1985). The percentage of replicate trees in which the associated taxa clustered together in the bootstrap test (1,000 replicates) are shown next to the branches. The evolutionary distances were computed using the Poisson correction method (Zuckerkandl et al., 1965) and are in the units of the number of amino acid substitutions per site. The analysis involved 106 amino acid sequences. All positions with $<50 \%$ site coverage were eliminated. That is, fewer than $50 \%$ alignment gaps, missing data, and ambiguous bases were allowed at any position. There were a total of 196 positions in the final dataset. Evolutionary analyses were conducted in MEGA7 (Kumar et al., 2016). Clades with strong bootstrap support are numbered I-XXVII (Figure S2). Clades with red colored branches contain lipocalins detected in adult ticks feeding for 5 days (Beaufays et al., 2008a); these are marked with a red symbol. Branches of green color contain sequences that are upregulated at time zero when ticks are infected with TBEV; these are marked with a green square. Branches of turquoise color contain sequences that are upregulated at $1 \mathrm{~h}$ in TBEV-infected ticks; these are marked with a turquoise triangle.

\section{PCR Validation}

Pooled tick RNA was converted into cDNA using a REPLI-g WTA Single Cell kit (Qiagen) in accordance with the kit 
TABLE 1 | Regulation of individual contigs and associated proteins in response to TBEV infection in salivary glands of unfed /xodes ricinus ticks and at one and three hours of feeding organized by category, showing proteases and miscellaneous genes.

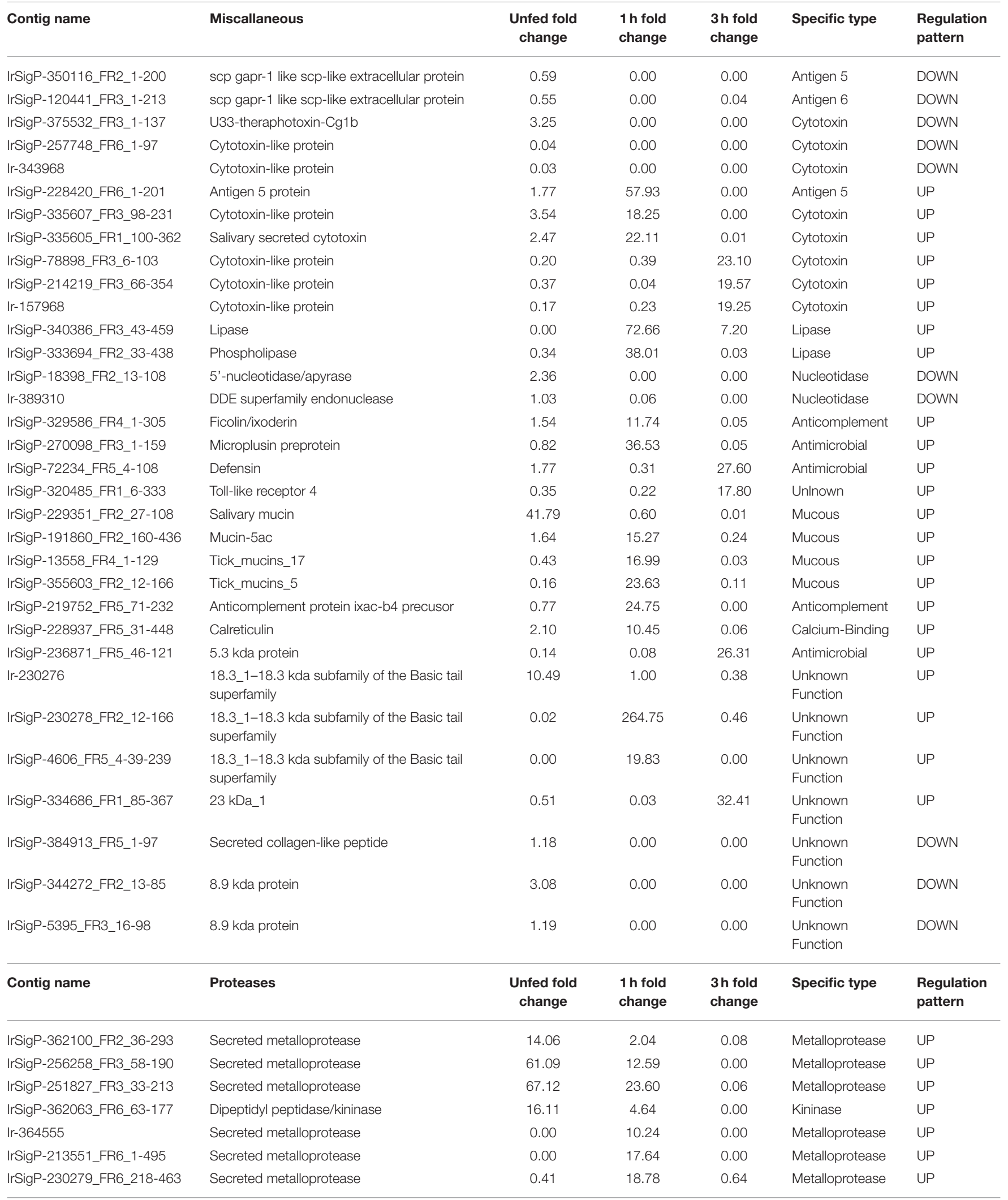


TABLE 1 | Continued

\begin{tabular}{|c|c|c|c|c|c|c|}
\hline Contig name & Proteases & $\begin{array}{l}\text { Unfed fold } \\
\text { change }\end{array}$ & $\begin{array}{l}1 \mathrm{~h} \text { fold } \\
\text { change }\end{array}$ & $\begin{array}{l}3 \mathrm{~h} \text { fold } \\
\text { change }\end{array}$ & Specific type & $\begin{array}{l}\text { Regulation } \\
\text { pattern }\end{array}$ \\
\hline IrSigP-317111_FR6_39-280 & Secreted metalloprotease & 0.15 & 27.38 & 0.00 & Metalloprotease & UP \\
\hline IrSigP-213340_FR5_1-177 & Secreted metalloprotease & 2.05 & 27.28 & 0.08 & Metalloprotease & UP \\
\hline IrSigP-7165_FR5_1-150 & Secreted metalloprotease & 0.08 & 42.47 & 0.00 & Metalloprotease & UP \\
\hline IrSigP-213336_FR3_28-166 & Secreted metalloprotease & 3.03 & 42.20 & 0.00 & Metalloprotease & UP \\
\hline IrSigP-317110_FR2_1-150 & Secreted metalloprotease & 0.02 & 86.16 & 0.01 & Metalloprotease & UP \\
\hline IrSigP-218287_FR3_180-401 & Secreted metalloprotease & 0.00 & 110.78 & 0.43 & Metalloprotease & UP \\
\hline IrSigP-195061_FR1_23-118 & Metalloprotease & 0.90 & 11.21 & 0.01 & Metalloprotease & UP \\
\hline IrSigP-349107_FR2_284-526 & Metalloprotease & 1.69 & 14.64 & 0.10 & Metalloprotease & UP \\
\hline IrSigP-251870_FR1_13-811 & M13 family peptidase & 0.83 & 23.95 & 0.00 & $\begin{array}{l}\text { Neprilysin-Like } \\
\text { Protease }\end{array}$ & UP \\
\hline IrSigP-364331_FR3_106-370 & Cathepsin B-like cysteine protease form 1 & 0.06 & 18.88 & 0.00 & $\begin{array}{l}\text { Cysteine } \\
\text { Protease }\end{array}$ & UP \\
\hline Ir-240260 & Neutral endopeptidase-like protein & 2.30 & 13.22 & 0.03 & $\begin{array}{l}\text { Neprilysin-Like } \\
\text { Protease }\end{array}$ & UP \\
\hline IrSigP-343497_FR6_11-110 & Serin protease & 0.30 & 10.81 & 0.00 & Serine Protease & UP \\
\hline IrSigP-371761_FR5_22-194 & Typsin-like serin protease & 1.18 & 32.41 & 0.15 & Serine Protease & UP \\
\hline IrSigP-305695_FR2_95-261 & Typsin-like serin protease & 1.79 & 67.28 & 2.19 & Serine Protease & UP \\
\hline IrSigP-365277_FR5_14-279 & Typsin-like serin protease & 1.90 & 11.94 & 0.03 & Serine Protease & UP \\
\hline IrSigP-305694_FR4_27-296 & Typsin-like serin protease & 1.69 & 49.62 & 0.64 & Serine Protease & UP \\
\hline IrSigP-371762_FR1_7-330 & Typsin-like serin protease & 1.61 & 102.54 & 0.45 & Serine Protease & UP \\
\hline IrSigP-299712_FR2_1-282 & Secreted metalloprotease & 0.45 & 0.95 & 18.61 & Metalloprotease & UP \\
\hline IrSigP-212742_FR1_38-533 & Secreted metalloprotease & 0.50 & 0.24 & 10.11 & Metalloprotease & UP \\
\hline Ir-263403 & Peptidase family M28 & 0.35 & 0.03 & 11.55 & Metalloprotease & UP \\
\hline IrSigP-321314_FR5_72-813 & M13 family peptidase & 0.75 & 0.11 & 11.56 & $\begin{array}{l}\text { Neprilysin-Like } \\
\text { Protease }\end{array}$ & UP \\
\hline Ir-66057 & Peptidase family m13 & 0.26 & 0.06 & 13.96 & $\begin{array}{l}\text { Neprilysin-Like } \\
\text { Protease }\end{array}$ & UP \\
\hline IrSigP-9195_FR5_47-292 & Secreted metalloprotease & 6.44 & 0.06 & 0.02 & Metalloprotease & DOWN \\
\hline
\end{tabular}

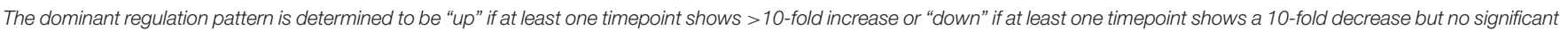
upregulation is observed.

instructions for purified total RNA. In brief, the kit generates cDNA from the RNA template, ligates the resulting cDNA into high molecular weight DNA molecules, and finally copies the ligated DNA using a proofreading DNA polymerase. This type of kit is more efficient than others and generates a larger quantity of cDNA for qPCR. The product of the kit was diluted 1:100 with molecular-grade water.

Targets of interest were identified based on the NGS data. The targets were required to be statistically significant at all three timepoints, to have an FPKM of ten or greater, and a change in expression of at least ten in at least one timepoint. Nineteen genes were selected from this group based on number of reads and the presence of sequence indicators representing secretion into the saliva.

Primers were designed based on sequencing data and ordered from Integrated DNA technologies. The sequences of these primers are listed in Table S1. They were reconstituted with molecular grade water and diluted to $10 \mu \mathrm{M}$. These samples were run in duplicate on a Biorad IQ5 machine using $10 \mu \mathrm{L}$ Biorad iTaq Universal SYBR Green Mix, $0.6 \mu \mathrm{L}$ of forward primer, 0.6 $\mu \mathrm{L}$ of reverse primer, $3.3 \mu \mathrm{L}$ molecular grade water, and $2 \mu \mathrm{L}$ diluted cDNA per sample. The thermal cycle included $50^{\circ} \mathrm{C}$ for $10 \mathrm{~min}, 95^{\circ} \mathrm{C}$ for $3 \mathrm{~min}$, and then 45 cycles of $95^{\circ} \mathrm{C}$ for $15 \mathrm{~s}$ and $60^{\circ} \mathrm{C}$ for $30 \mathrm{~s}$ during which the samples were observed by the machine. After completion, melt curves were automatically generated, beginning with treatment at $95^{\circ} \mathrm{C}$ for $1 \mathrm{~min}$ followed by $55^{\circ} \mathrm{C}$ for $1 \mathrm{~min}$ and 81 observation cycles beginning at $55^{\circ} \mathrm{C}$. The final results were baseline-normalized at an absorbance 100 .

The Ct values between the technical duplicates were averaged, and changes in expression were calculated (Figure S1) using the $\Delta \Delta \mathrm{Ct}$ method, normalizing against the tick ribosomal S4 signal (Cabezas-Cruz et al., 2016).

\section{RESULTS AND DISCUSSION}

To understand the differential response of the I. ricinus salivary gland transcriptome during early stages of TBEV transmission, ticks were inoculated with TBEV by coxal microinjection 21 days prior to being fed on mice. This technique for infection is allowed for the generation of infected ticks with a high rate of success and has been widely accepted (Labuda et al., 2006; Slovák et al., 2014; 
TABLE 2 | Regulation of individual contigs and associated proteins in response to TBEV infection in salivary glands of unfed /xodes ricinus ticks and at one and three hours of feeding organized by category, showing lipocalins, Kunitz-domain proteins, and glycine-rich family proteins

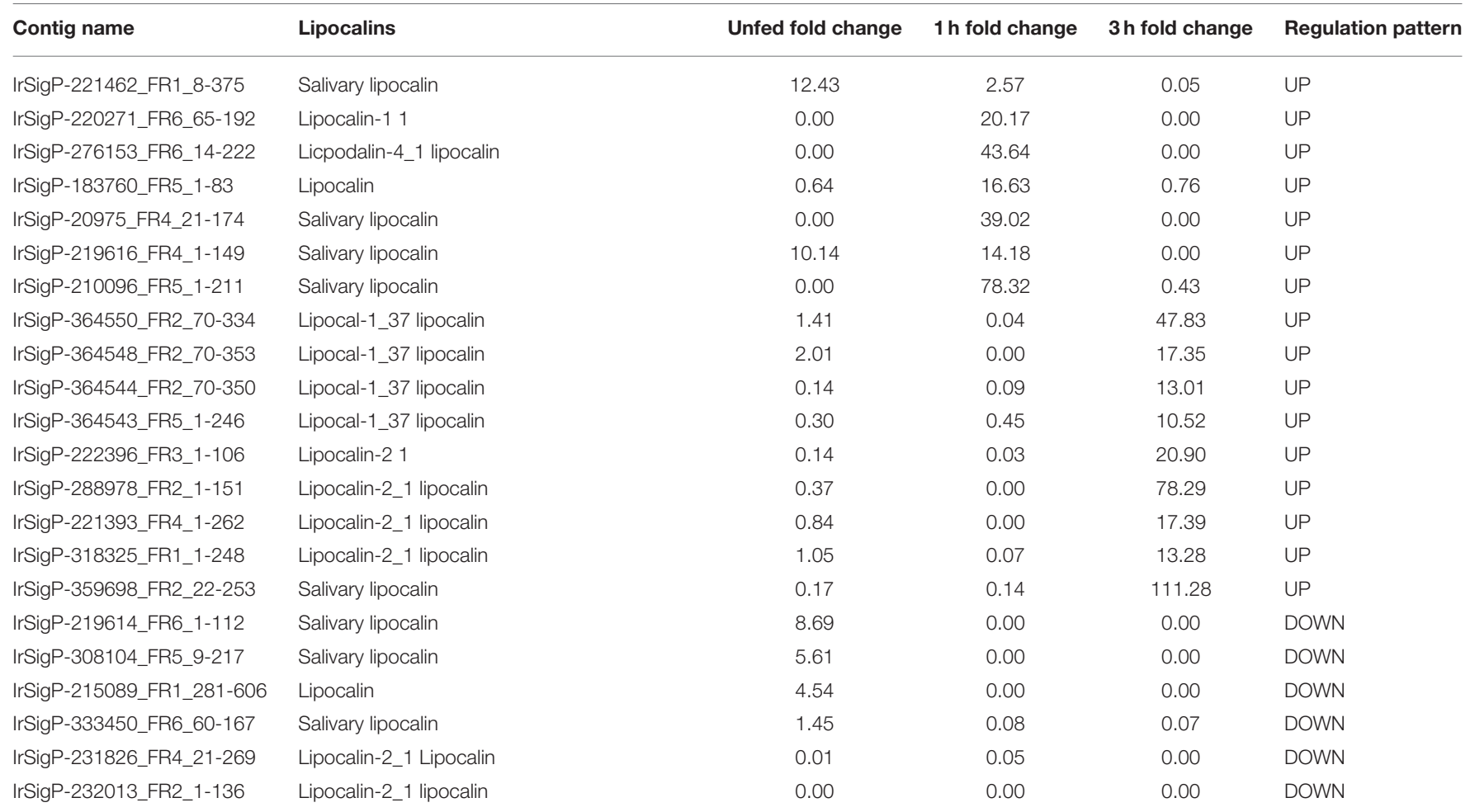

\begin{tabular}{|c|c|c|c|c|c|}
\hline Contig name & Kunitz-Domain proteins & Unfed fold change & $1 \mathrm{~h}$ fold change & $3 \mathrm{~h}$ fold change & Regulation pattern \\
\hline IrSigP-233783_FR4_27-108 & Salivary kunitz domain protein & 12.26 & 1.89 & 0.02 & UP \\
\hline IrSigP-267369_FR6_1-92 & Salivary kunitz domain protein & 54.15 & 0.00 & 0.00 & UP \\
\hline IrSigP-247833_FR5_76-171 & Tick kunitz 46 & 13.40 & 1.16 & 0.03 & UP \\
\hline IrSigP-138775_FR4_1-100 & Tick_Kunitz_56 & 12.02 & 0.00 & 0.00 & UP \\
\hline IrSigP-353563_FR5_1-97 & Serine proteinase inhibitor (KU family) & 0.00 & 61.68 & 0.00 & UP \\
\hline IrSigP-5685_FR5_27-338 & Salivary kunitz domain protein & 0.59 & 138.37 & 0.43 & UP \\
\hline IrSigP-300370_FR3_1-184 & Tick kunitz 38 & 1.80 & 10.64 & 0.03 & UP \\
\hline IrSigP-286607_FR3_15-359 & Tick_Kunitz_134 & 1.67 & 13.77 & 0.05 & UP \\
\hline IrSigP-389808_FR1_1-86 & Tick_Kunitz_44 & 0.80 & 48.52 & 0.04 & UP \\
\hline Contig name & Glycine-Rick (Cement) proteins & Unfed fold change & $1 \mathrm{~h}$ fold change & $3 \mathrm{~h}$ fold change & Regulation pattern \\
\hline $\mid r-12879$ & GRP-2_441 Glycine rich family & 0.00 & 14.03 & 0.00 & UP \\
\hline Ir-216707 & GRP-2_441 Glycine rich family & 2.28 & 19.63 & 0.02 & UP \\
\hline IrSigP-8569_FR6_25-160 & GRP-2_441 Glycine rich family & 2.26 & 46.52 & 0.02 & UP \\
\hline |r-357614 & GRP-2_441 Glycine rich family & 3.54 & 63.88 & 0.00 & UP \\
\hline IrSigP-345293_FR5_35-172 & GRP-2_441 Glycine rich family & 0.00 & 83.52 & 0.46 & UP \\
\hline IrSigP-361126_FR6_1-133 & GRP-2_441 Glycine rich family & 1.80 & 105.26 & 0.00 & UP \\
\hline IrSigP-363306_FR5_53-179 & GRP-2_441 Glycine rich family & 0.18 & 151.07 & 0.47 & UP \\
\hline
\end{tabular}


TABLE 2 | Continued

\begin{tabular}{|c|c|c|c|c|c|}
\hline Contig name & Glycine-Rick (Cement) proteins & Unfed fold change & $1 \mathrm{~h}$ fold change & $3 \mathrm{~h}$ fold change & Regulation pattern \\
\hline IrSigP-347965_FR2_15-168 & GRP-2_441 Glycine rich family & 0.02 & 168.29 & 0.11 & UP \\
\hline IrSigP-209173_FR2_40-179 & GRP-2_449 Glycine rich family & 0.00 & 20.34 & 0.64 & UP \\
\hline IrSigP-20709_FR3_56-211 & GRP-2_590 Glycine rich family & 0.49 & 0.05 & 23.37 & UP \\
\hline IrSigP-354603_FR4_25-133 & GRP-2_471 Glycine rich family & 1.91 & 0.00 & 0.00 & DOWN \\
\hline
\end{tabular}

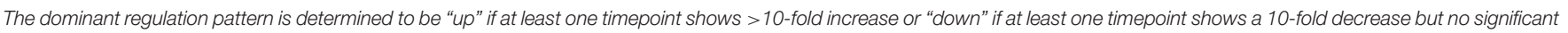
upregulation is observed.

Thangamani et al., 2017). Cohorts of infected and non-infected ticks were then allowed to feed on mice. At times $1 \mathrm{~h}$ and $3 \mathrm{~h}$ post attachment, ticks were removed from the mice for salivary gland dissection followed by RNA extraction. The RNAs were then sequenced and analyzed.

\section{Distribution of Changes in Gene Expression}

Several classes of transcripts with significant salivary functions were identified as either up- or down-regulated in response to infection with TBEV. The numbers of genes with significant changes in regulation in each class in unfed, $1 \mathrm{~h}$ fed, and $3 \mathrm{~h}$ fed ticks are summarized in Table 3. In unfed ticks, only 33 genes were observed to be significantly upregulated in response to infection, as opposed to 214 after $1 \mathrm{~h}$ of tick attachment and 138 after $3 \mathrm{~h}$. Comparing infected ticks to uninfected ticks, the number of downregulated genes in unfed infected ticks was 104 , as opposed to 138 after $1 \mathrm{~h}$ of feeding and 174 after $3 \mathrm{~h}$. This suggests that the strongest viral-induced changes to salivary expression occur after $1 \mathrm{~h}$ of feeding. This ability to modulate expression at a specific temporal point suggests that the virus has a direct and specific effect on gene regulation during the feeding process as opposed to a generic, global change in response to infection.

The changes in expression skew heavily toward uncharacterized genes whose function is unknown (Figure 1), which make up between 58 and $84 \%$ of up- or down-regulated genes in each category. Proteases, serine protease inhibitors, and lipocalins tended to be the dominant functionally identifiable classes of genes, although their proportion of the total of upor down-regulated genes varies substantially by timepoint. This was especially notable in unfed ticks, where $18 \%$ of the upregulated genes were identified as serpins or Kunitzdomain proteins, and $12 \%$ were proteases and metalloproteases. This proportion decreased during feeding so that after $3 \mathrm{~h}$, lipocalins were more prevalently upregulated (7\%) while serine protease inhibitors were not altered, and protease involvement had dropped to $4 \%$ of the genes. The opposite pattern is observed in downregulated genes: although a group of lipocalins was observed to be downregulated relatively consistently $(5,9$, and $6 \%$ at unfed, 1 and $3 \mathrm{~h}$ timepoint, respectively), the proportion of downregulated proteases and serpin/Kunitz-domain proteins increased.

The expression of individual genes was also found to be remarkably inconstant across the three timepoints. None of the identified genes were found to be significantly upregulated at more than one timepoint. Many genes would often be upregulated at some points and downregulated at others (Tables 1, 2 and Figures 2, 3). These results indicate that viral interaction with the transcriptional regulation of salivary proteins is a highly dynamic process. Different sets of proteins are expressed at each individual timepoint in accordance with the stage of tick feeding; these, in turn, have different effects on the host immune response to both the tick and the virus it introduces.

\section{IMPLICATIONS OF INDIVIDUAL PROTEIN CLASSES ON VIRAL BEHAVIOR AND TRANSMISSION}

\section{Serine Protease Inhibitors}

Serine protease inhibitors of the Kunitz family comprise a broad class of enzyme inhibitors that generally serve a regulatory purpose. In some animals, however, these inhibitors evolved to serve as venom (Mourao and Schwartz, 2013). For arachnids, Kunitz-domain-containing proteins either interfere with ion channel activity (Chen et al., 2012; Dai et al., 2012; Valdes and Moal, 2014; Santibañez-Lopez et al., 2018) or inhibit critical host proteins (Decrem et al., 2008; Bronsoms et al., 2011; Andreotti et al., 2012; Louw et al., 2013; Zhang et al., 2017). Tick saliva is essentially a form of venom evolved to facilitate bloodfeeding, and although Ixodes spp. ticks retain some of their ion channel-manipulating Kunitz-domain peptides (Dai et al., 2012; Valdes and Moal, 2014), they largely fall within the enzyme inhibitor group.

Kunitz-domain serine protease inhibitors are known to inhibit coagulation (Dai et al., 2012; Chen et al., 2013; Louw et al., 2013; Assumpção et al., 2015; Zhang et al., 2017), angiogenesis (Drewes et al., 2012; Soares et al., 2016), and may reduce inflammation (Bronsoms et al., 2011). Their primary purpose is to ensure successful feeding by inhibiting blood coagulation. Multiple inhibitors are expressed to target several proteins involved in the coagulation cascade.

A steady supply of blood is required for a tick to continuously salivate. Unlike spiders, ticks have no equivalent to a venom sack and cannot synthesize and store substantial quantities of saliva in advance. It is instead produced in real-time during feeding, with the necessary fluid being provided as the bloodmeal is concentrated in the midgut. Viral transmission requires this continuous flow of outgoing fluid both as a vehicle for entry into the host and because the immunomodulatory portion of 
TABLE 3 | Numbers of genes up and downegulated in response to TBEV infection in salivary glands of unfed Ixodes ricinus ticks and at one and three hours of feeding, categorized by predicted functional class.

\begin{tabular}{|c|c|c|c|c|c|c|}
\hline \multirow[t]{2}{*}{ Class } & \multicolumn{6}{|c|}{ Number of genes } \\
\hline & $\begin{array}{l}\text { Upregulated } \\
\text { (Unfed) }\end{array}$ & $\begin{array}{l}\text { Downregulated } \\
\text { (Unfed) }\end{array}$ & $\begin{array}{l}\text { Upregulated } \\
\text { (1 h feeding) }\end{array}$ & $\begin{array}{l}\text { Downregulated } \\
\text { (1 } \mathrm{h} \text { feeding) }\end{array}$ & $\begin{array}{l}\text { Upregulated } \\
\text { (3h feeding) }\end{array}$ & $\begin{array}{c}\text { Downregulated } \\
\text { (3 h feeding) }\end{array}$ \\
\hline Cytotoxin/Antigen 5 & 1 & 2 & 3 & 6 & 3 & 9 \\
\hline Digestive & 0 & 1 & 2 & 2 & 0 & 3 \\
\hline Glycine-Rich & 0 & 4 & 9 & 2 & 1 & 6 \\
\hline Immune & 0 & 0 & 2 & 0 & 2 & 2 \\
\hline Lipocalin & 1 & 5 & 6 & 13 & 9 & 9 \\
\hline Mucin & 1 & 0 & 3 & 0 & 0 & 2 \\
\hline Other/Unknown & 1 & 2 & 4 & 5 & 2 & 6 \\
\hline Protease & 4 & 6 & 20 & 3 & 5 & 18 \\
\hline Kunitz-Domain & 6 & 2 & 6 & 5 & 0 & 13 \\
\hline Uncategorized & 19 & 82 & 159 & 102 & 116 & 106 \\
\hline Total & 33 & 104 & 214 & 138 & 138 & 174 \\
\hline
\end{tabular}

the saliva generates a microenvironment within the host that enhances viral infection (Thangamani et al., 2017).

As such, viral infection benefits from maintenance and enhancement of the anticoagulation system during the early stages of infection. Six Kunitz-type inhibitors and an arenetoxin analog (a probable trypsin inhibitor) were found to be upregulated in unfed ticks, with an average fold change of twenty and including one transcript that demonstrated a 54fold increase. These represent a small amount of initial saliva prepared for an immediate and robust anticoagulant effect to facilitate more rapid early feeding. This group is not substantially upregulated at $1 \mathrm{~h}$ of feeding but shows downregulation when detected by $3 \mathrm{~h}$ with a fold change of $0.02-0.03$.

Six additional Kunitz-type inhibitors were upregulated by infection after $1 \mathrm{~h}$ of feeding. The level of upregulation was more diverse at this timepoint than in unfed ticks, with three values having a fold change between 10 and 20, one having a change of 49-fold, one with a change of 62 -fold, and one with a change of 138 -fold. These contigs were not found upregulated at any other timepoint, and several were reduced at $3 \mathrm{~h}$ of feeding, including a change from 11 to 0.03 fold, 14 to 0.05 fold, and 49 to 0.04 fold.

At $3 \mathrm{~h}$ of feeding, the overall trend was a decreased expression of Kunitz-like inhibitors in response to viral infection, with no upregulated genes observed. Downregulation was not observed at any timepoint apart from $3 \mathrm{~h}$ in all but three genes, two of which were downregulated at 1 (0.008 and 0.03 fold) and one downregulated in uninfected ticks ( 0.02 fold). This downregulation continued for $3 \mathrm{~h}$.

By $3 \mathrm{~h}$, the ticks have already transmitted the infectious dose of the virus (Hermance and Thangamani, 2018) and have already imbibed a small supply of fluid from the host. Forced downregulation of the anticoagulant response interferes with feeding and may serve as a mechanism to trigger additional salivation (using already-consumed blood as a fluid source) as the tick attempts to compensate for increasingly poor fluidity of its bloodmeal. This process, in turn, forces the tick to inject more virus and immune-modulatory factors into the host. It may also lead to tick dislodgement of the host.

\section{Lipocalins}

Lipocalins are proteins that bind to small organic molecules. Within the context of tick saliva, lipocalins are used to sequester histamine (Paesen et al., 1999; Sangamnatdej et al., 2002; DiazMartin et al., 2011; Valdes et al., 2016; Wang et al., 2016; Neelakanta et al., 2018), serotonin (Xu et al., 2013), leukotrienes (Beaufays et al., 2008a,b; Mans and Ribeiro, 2009), and other signaling molecules to reduce the anti-tick inflammatory response. A critical role of salivary lipocalins is to reduce itch and pain response resulting from histamine and serotonin signaling. This prevents from a scratching response of the host that could potentially damage the tick. Additionally, some salivary lipocalins have also often evolved secondary functions including anticoagulant activity (Mans and Ribeiro, 2008), as well as the ability to modulate the behavior of leukocytes (Rodriguez-Valle et al., 2013) and dendritic cells (Preston et al., 2013).

Our data show that lipocalins are differentially expressed at different timepoints during early phases of tick feeding, and the presence of TBEV causes both upregulation and downregulation of particular lipocalins. Unlike Kunitz-domain inhibitors, which tended toward early upregulation and later downregulation, lipocalins were more heavily upregulated at later timepoints but showed less significant changes in unfed ticks. Only two contigs showed upregulation in unfed, infected ticks with fold-changes of 12 and 10 compared to uninfected controls. By $1 \mathrm{~h}$ of feeding, six genes were upregulated in infected ticks vs. controls. This includes one of the genes that was upregulated in unfed ticks, which changed from 10 - to a 14-fold increase. Other genes showed fold changes of $17,20,44$, and 78 , which represents a substantial change in several lipocalins.

At $3 \mathrm{~h}$, nine genes were upregulated in infected ticks compared to uninfected ones. Six of these were between 10 and 21-fold. The remaining three showed fold changes of 48, 78, and 111 . Although this trend is similar to what was observed at $1 \mathrm{~h}$, the 


\begin{tabular}{|c|c|c|c|c|}
\hline Contig Name & Miscellaneous Proteins & Unfed & $1 \mathrm{~h}$ & $3 \mathrm{~h}$ \\
\hline IrSigP-350116_FR2_1-200 & scp gapr-1 like scp-like extracellular protein & & & \\
\hline IrSigP-120441_FR3_1-213 & scp gapr-1 like scp-like extracellular protein & & & \\
\hline IrSigP-375532_FR3_1-137 & U33-theraphotoxin-Cg1b & & & \\
\hline IrSigP-257748 FR6 ${ }^{-} 1-97$ & cytotoxin-like protein & & & \\
\hline Ir-343968 & cytotoxin-like protein & & & \\
\hline IrSigP-228420_FR6_1-201 & antigen 5 protein & & & \\
\hline IrSigP-335607_FR3_98-231 & cytotoxin-like protein & & & \\
\hline IrSigP-335605_FR1_100-362 & salivary secreted cytotoxin & & & \\
\hline IrSigP-78898_FR3_-̄-103 & cytotoxin-like protein & & & \\
\hline IrSigP-214219̄_FR3 $366-354$ & cytotoxin-like protein & & & \\
\hline Ir-157968 & cytotoxin-like protein & & & \\
\hline IrSigP-340386_FR3_43-459 & lipase & & & \\
\hline IrSigP-333694_FR2_33-438 & phospholipase & & & \\
\hline IrSigP-18398_FR2_13-108 & 5'-nucleotidase/apyrase & & & \\
\hline Ir-389310 & DDE superfamily endonuclease & & & \\
\hline IrSigP-329586_FR4_1-305 & ficolin/ixoderin & & & \\
\hline IrSigP-270098_FR3_1-159 & microplusin preprotein & & & \\
\hline IrSigP-72234_FR5_4-108 & defensin & & & \\
\hline IrSigP-320485_FR1_6-333 & toll-like receptor 4 & & & \\
\hline IrSigP-229351_FR2_27-180 & salivary mucin & & & \\
\hline IrSigP-191860_FR2_160-436 & mucin-5ac & & & \\
\hline IrSigP-13558_FR4_-̄1-129 & Tick_mucins_17 & & & \\
\hline IrSigP-355603_FR2_12-166 & Tick_mucins_2 & & & \\
\hline IrSigP-219752_FR5_71-232 & anticomplemént protein ixac-b4 precursor & & & \\
\hline IrSigP-228937_FR5_31-448 & Calreticulin & & & \\
\hline IrSigP-236871_FR5_46-121 & 5.3 kda protein & & & \\
\hline Ir-230276 & 18.3_1 $18.3 \mathrm{kda}$ subfamily of the Basic tail superfamily & & & \\
\hline IrSigP-230278_FR2_12-166 & 18.3_1 - $18.3 \mathrm{kda}$ subfamily of the Basic tail superfamily & & & \\
\hline IrSigP-4606_FR4_39-239 & 18.3 -1 $18.3 \mathrm{kda}$ subfamily of the Basic tail superfamily & & & \\
\hline IrSigP-33468̄6_FR'1_85-367 & 23kDa_1 & & & \\
\hline IrSigP-384913_FR5_1-97 & secreted collagen-like peptide & & & \\
\hline IrSigP-344272_FR2_13-85 & 8.9 kda protein & & & \\
\hline IrSigP-5395_FR3_16-98 & 8.9 kda protein & & & \\
\hline
\end{tabular}

FIGURE 2 | Heatmaps of several categories of genes identified in salivary glands of /xodes ricinus ticks as up or down regulated in response to TBEV infection, including miscellaneous genes (cytotoxins, anti-microbial genes, and mucins). Regulation is generally most influenced at a single timepoint, often as the inverse of the pattern seen in other timepoints. Expression in each category is also not consistent between proteins, indicating that expression of each individual gene is not equivalent, and under complex regulatory control.

genes expressed are different at $3 \mathrm{~h}$. Many of these 3-h genes were actually downregulated at $1 \mathrm{~h}$, including 0.04 ( 48 at $3 \mathrm{~h}$ ), 0.08 (13 at $3 \mathrm{~h}), 0.03$ (21 at $3 \mathrm{~h}), 0.07$ (13 at $3 \mathrm{~h}$ ), and one as low as 0.0004 which became 17 at $3 \mathrm{~h}$.

Additionally, six lipocalins were not found to be upregulated in infected ticks, but significantly downregulated compared to control ticks in at least one group.

Since $I$. ricinus lipocalins have been segregated into distinct phylogenetic groups (Beaufays et al., 2008a), we constructed a phylogram using the lipocalin sequences found in this study plus the lipocalin sequences described in Beaufays et al. (2008a), which were derived from transcripts of adult ticks feeding for 5 days. The resulting phylogram, made from 106 sequences, is complex, producing 27 clades with strong bootstrap support (Figure S2). Interestingly, the sequences that were upregulated at zero or $1 \mathrm{~h}$ are in different clades. All the sequences derived from 5 day feeding ticks inhabit clades that are not shared by those upregulated by TBEV. This is not surprising, since ticks switch their sialomes with time (Karim and Ribeiro, 2015; Perner et al., 2018), possibly as an immune evasion mechanism. Thus, the different clades may represent proteins with different functions, or with different antigenic character. Whether the different clades containing TBEV upregulated lipocalins at zero and $1 \mathrm{~h}$ represent different lipocalin functions or different antigenicity cannot be resolved.

From the perspective of viral infection, these results are complex. It has been previously demonstrated that the presence of TBEV causes an increased inflammatory reaction during a tick-bite (Thangamani et al., 2017). Viral transmission benefits from a pro-inflammatory 


\begin{tabular}{|c|c|c|c|c|c|c|c|c|c|}
\hline Contig Name & Lipocalins & Unfed & $1 \mathrm{~h}$ & $3 \mathrm{~h}$ & Contig Name & Proteases & Unfed & $1 \mathrm{~h}$ & $3 \mathrm{~h}$ \\
\hline IrSigP-221462_FR1_8-375 & salivary lipocalin & & & & IrSigP-362100_FR2_36-293 & Secreted metalloprotease & & & \\
\hline IrSigP-220271_FR6_65-192 & lipocal-11 & & & & IrSigP-256258_FR3_58-190 & Secreted metalloprotease & & & \\
\hline IrSigP-276153_FR6_14-222 & Licpodalin-4_1 lipocalin & & & & IrSigP-251827_FR3_33-213 & Secreted metalloprotease & & & \\
\hline IrSigP-183760_FR5_1-83 & lipocalin & & & & IrSigP-362063_FR6_63-177 & dipeptidyl peptidase/kininase & & & \\
\hline IrSigP-20975_FR4_21-174 & salivary lipocalin & & & & Ir-364555 & Secreted metalloprotease & & & \\
\hline IrSigP-219616_FR4_1-149 & salivary lipocalin & & & & IrSigP-213551_FR6_1-495 & Secreted metalloprotease & & & \\
\hline IrSigP-210096_FR5_1-211 & salivary lipocalin & & & & IrSigP-230279_FR6_218-463 & Secreted metalloprotease & & & \\
\hline IrSigP-364550_FR2_70-334 & Lipocal-1_37 lipocalin & & & & IrSigP-317111_FR6_39-280 & Secreted metalloprotease & & & \\
\hline IrSigP-364548_FR2_70-353 & \multirow{2}{*}{$\begin{array}{l}\text { Lipocal-1_37 lipocalin } \\
\text { Lipocal-1_37 lipocalin }\end{array}$} & & & & IrSigP-213340_FR5_1-177 & Secreted metalloprotease & & & \\
\hline IrSigP-364544_FR2_70-350 & & & & & IrSigP-7165_FR5_1-210 & Secreted metalloprotease & & & \\
\hline IrSigP-364543_FR5_1-246 & Lipocal-1_37 lipocalin & & & & IrSigP-213336_FR3_28-166 & Secreted metalloprotease & & & \\
\hline IrSigP-222396_FR3_1-106 & lipocalin- $\overline{2} 1$ & & & & IrSigP-317110_FR2_1-150 & Secreted metalloprotease & & & \\
\hline IrSigP-288978_FR2_1-151 & Lipocalin-2_1 lipocalin & & & & IrSigP-218287_FR3_180-401 & Secreted metalloprotease & & & \\
\hline IrSigP-221393_FR4_1-262 & Lipocalin-2_1 lipocalin & & & & IrSigP-195061_FR1_23-118 & metalloprotease & & & \\
\hline IrSigP-318325_FR1_1-248 & Lipocalin-2_1 lipocalin & & & & IrSigP-349107_FR2_284-526 & metalloprotease & & & \\
\hline IrSigP-359698_FR2_22-253 & salivary lipocalin & & & & IrSigP-251870_FR1_13-811 & M13 family peptidase & & & \\
\hline IrSigP-219614_FR6_1-112 & salivary lipocalin & & & & IrSigP-364331_FR3_106-370 & Cathepsin B-like cysteine protease form 1 & & & \\
\hline IrSigP-308104_FR5_9-217 & salivary lipocalin & & & & Ir-240260 & neutral endopeptidase-like protein & & & \\
\hline IrSigP-215089_FR1_281-606 & lipocalin & & & & IrSigP-343497_FR6_11-110 & serine protease & & & \\
\hline IrSigP-333450_FR6_60-167 & salivary lipocalin & & & & IrSigP-371761_FR5_22-194 & trypsin-like serine protease & & & \\
\hline IrSigP-231826_FR4_21-269 & \multirow{2}{*}{\multicolumn{2}{|c|}{$\begin{array}{l}\text { Lipocalin-2_1 lipocalin } \\
\text { Lipocalin-2_1 lipocalin }\end{array}$}} & & & IrSigP-305695_FR2_95-261 & trypsin-like serine protease & & & \\
\hline IrSigP-232013_FR2_1-136 & & & & & IrSigP-365277_FR5_14-279 & trypsin-like serine protease & & & \\
\hline & \multicolumn{2}{|l|}{ Lipocalin-2_1 lipocalin } & & & IrSigP-305694_FR4_27-296 & trypsin-like serine protease & & & \\
\hline Contig Name & Kunitz-Domain Proteins & Unfed & $1 \mathrm{~h}$ & $3 \mathrm{~h}$ & IrSigP-371762_FR1_7-330 & trypsin-like serine protease & & & \\
\hline & BPTI/Kunitz family of serine protease & & & & $\begin{array}{l}\text { IrSigP-299712_FR2_1-282 } \\
\text { IrSigP-212742_FR1 38-533 }\end{array}$ & $\begin{array}{l}\text { Secreted metalloprotease } \\
\text { Secreted metalloprotease }\end{array}$ & & & \\
\hline IrSigP-196594_FR3_14-286 & inhibitors & & & & Ir-263403 & Peptidase family M28 & & & \\
\hline IrSigP-233783_FR4_27-108 & salivary Kunitz domain protein & & & & IrSigP-321314_FR5_72-813 & M13 family peptidase & & & \\
\hline IrSigP-194429_FR6_23-125 & salivary Kunitz domain protein & & & & Ir-66057 & peptidase family $\mathrm{m} 13$ & & & \\
\hline IrSigP-267369_FR6_1-92 & salivary Kunitz domain protein & & & & IrSigP-9195_FR5_47-292 & Secreted metalloprotease & & & \\
\hline IrSigP-247833_FR5_76-171 & tick Kunitz 46 & & & & & & & & \\
\hline IrSigP-138775_FR4_1-100 & tick_Kunitz_56 & & & & & & & & \\
\hline IrSigP-226572_FR5_1-141 & u3-aranetoxin-ce1a & & & & Contig Name & Glycine-Rich Proteins & Unfed & $1 \mathrm{~h}$ & $3 \mathrm{~h}$ \\
\hline IrSigP-353563_FR5_1-97 & Serine proteinase inhibitor (KU family) & & & & Ir-12879 & GRP-2_441 Glycine rich family & & & \\
\hline IrSigP-5685_FR5_27-338 & salivary Kunitz domain protein & & & & II-216707 & GRP-2_441 Glycine rich family & & & \\
\hline $\begin{array}{l}\text { IrSigP-300370_FR3_1-184 } \\
\text { IrSigP-286607-FR3 } 15-359\end{array}$ & $\begin{array}{l}\text { tick Kunitz } 38 \\
\text { tick Kunitz } 134\end{array}$ & & & & IISigP-8569_FR6_25-160 & GRP-2_441 Glycine rich family & & & \\
\hline IrSigP-389808_FR1_1-86 & tick_Kunitz_44 & & & & |r-357614 & GRP-2_441 Glycine rich family & & & \\
\hline IrSigP-362807_FR3_68-283 & tick_Kunitz_88 & & & & IrSigP-345293_FR5_35-172 & GRP-2_441 Glycine rich family & & & \\
\hline IrSigP-292668_FR3_1-93 & tick_Kunitz_43 & & & & IrSigP-361126_FR6_1-133 & GRP-2_441 Glycine rich family & & & \\
\hline IrSigP-249585_FR2_1-123 & tick_Kunitz 53 & & & & IrSigP-363306_FR5_53-179 & GRP-2_441 Glycine rich family & & & \\
\hline IrSigP-257404_FR5_1-105 & salivary Kunitz domain protein & & & & IrSigP-347965_FR2_15-168 & GRP-2_441 Glycine rich family & & & \\
\hline & & & & & IrSigP-209173_FR2_40-179 & GRP-2_449 Glycine rich family & & & \\
\hline & & & & & IrSigP-20709_FR3_56-211 & GRP-2_590 Glycine rich family & & & \\
\hline & Downregulated & & & & IrSigP-354603_FR4_25-133 & GRP-2_471 Glycine rich family & & & \\
\hline
\end{tabular}

FIGURE 3 | Heatmaps of several categories of genes identified in salivary glands of Ixodes ricinus ticks as up or down regulated in response to TBEV infection, including lipocalins, proteases, and protease inhibitors (largely Kunitz-type). Regulation is generally most influenced at a single timepoint, often as the inverse of the pattern seen in other timepoints. Expression in each category is also not consistent between proteins, indicating that expression of each individual gene is not equivalent and under complex regulatory control.

response (Hermance and Thangamani, 2018) by summoning macrophages that can subsequently be infected and used to disseminate the virus to other tissues. By reducing histamineand leukotriene-binding lipocalins, the host response to the saliva becomes more conducive to inflammation and consequently macrophage and neutrophil invasion. Additionally, modulating the behavior of dendritic cells and leukocytes by upregulated lipocalin expression has the overall effect of making the tissue less responsive to the presence of virus and thereby directing the brunt of the response against the tick. This altered expression increases the itch response, risking tick damage by host scratching. Since transmission of TBEV starts within a few minutes after tick attachment (Hermance and Thangamani, 2018), destruction of the tick does not outright prevent the transmission of virus, and in addition, pressure on the tick may in fact force the tick to partially regurgitate into the skin. Ticks that are unable to feed properly can dislodge themselves (Piesman, 1991) and attach to new hosts to attempt to complete the feeding, thus increasing virus transmission.

\section{Lectins}

Lectins are carbohydrate-binding proteins found in tick saliva. Their exact role is poorly understood (Vechtova et al., 2018).
Their function is thought to be immunomodulatory or antimicrobial (Sterba et al., 2011; Smith and Pal, 2014), although their agglutination property has also been linked to blood digestion in the midgut (Vechtova et al., 2018). It is possible that, as in the case of spiders, ticks begin some of their digestion process externally through saliva; in ticks, this would specifically occur within the blood-pool before ingestion. Ticks also actively modulate the lectin-mediated complement system, for example by the tick salivary lectin pathway inhibitor (TSLP1) (Hajdušek et al., 2013); the simultaneous production of ficolin-like lectins in tick saliva therefore appears counterintuitive unless it either fails to initiate the host complement cascade or acts as a direct competitor to host ficolin on lectin receptors.

Ixoderin, an analog of ficolin, was found to be upregulated in infected ticks at $1 \mathrm{~h}$ feeding (with a fold-change of 12) but downregulated compared to controls at the $3 \mathrm{~h}$ timepoint (foldchange 0.05). It is difficult to determine the exact function of this particular lectin within the context of TBEV infection, as it could either modulate the host response or be produced as a reaction to viral infection of the salivary gland. If the latter is true and the lectin targets glycosylated amino acids on the viral particles, it may serve as an adaptor to protect the virus from mammalian host response or to facilitate phagocytosis and 
infection of macrophages. Functional studies will be required to confirm if this viral/lectin interaction is common.

\section{Cystatins}

Cystatins serve several functions within ticks, mainly with regard to hemoglobin digestion (Chmelar et al., 2017). Like tick lectins, they are present in tick saliva and also modulate host responses. Some inhibit TNF- $\alpha$ and IL12 production from dendritic cells and decrease CD4+ proliferation (Chmelar et al., 2017). TBEV replication in dendritic cells is enhanced by suppression of IFN signaling induced by cystatin exposure (Lieskovska et al., 2015). Cystatins have been shown to interfere with IL1 $\beta$ and IL18 in macrophages during Anaplasma infection and promote TBEV infection by further altering signaling in the dendritic cells. Cystatins bias the immune reaction away from the Th1 type that would be most effective against viral or intracellular pathogens (Schonenmeyer et al., 2017).

In our experiments, only one cystatin was observed with altered expression, although it is not listed in the presented tables and figures due to being down-regulated at only one of the required two exclusion points. It showed a fold-change of 0 (indicating that it was not detected in infected ticks) in unfed ticks and at $1 \mathrm{~h}$, and then it showed a non-significant fold-change of 3 at $3 \mathrm{~h}$.

This is unexpected, as the presence of cystatin is thought to enhance TBEV infection, yet is reduced here. The cystatin detected may be one of a more diverse group, may serve an alternate function and experience different regulation, as the sequencing data cannot positively identify it as cystatin 2, the salivary version. In this case, it probably serves a regulatory function in the way cystatin 3 does in human cells.

\section{Glycine-Rich Proteins}

Upon attaching to a host, ticks begin to secrete a thick proteinaceous cement to better secure their mouthparts to the host skin. This cement is immunogenic, and though largely structural it has an impact on modulating host responses. Glycine-rich proteins are one of the cement's characteristic constituents (Hollmann et al., 2018).

The presence of glycine-rich proteins is critical to the transmission of TBEV (Labuda et al., 2006). Mice vaccinated against a particular glycine-rich protein proved resistant to TBEV transmitted by ticks. This vaccine was derived from a cement protein from Rhipicephalus appendiculatus, but proved crossprotective against proteins from $I$. ricinus, suggesting strong evolutionary conservation between the proteins as well as high homology of their respective immunogenic sites.

Our data show that most of the glycine-rich proteins identified were upregulated substantially at $1 \mathrm{~h}$ feeding in TBEV infected ticks. Of eleven genes detected, nine were upregulated. Three were found upregulated between 10 and 20 fold, three between 45 and 85 fold, and three higher than 100 fold. Only one gene was upregulated at $3 \mathrm{~h}$ with a fold change of 23 , and the genes that were upregulated at $1 \mathrm{~h}$ were either not significantly changed or slightly downregulated. This most likely corresponds to a shift in the stage of cement deposition, with the viral-altered portion of the cement being applied early in the feeding and therefore of high early immunological relevance. These results imply that the production of cement has either increased or its composition has been altered in favor of its glycine-rich component. This corresponds with our observation that the production of tick mucin, another component of tick cement, was upregulated in response to infection at the 1 -h timepoint. Of four mucin genes detected, three were upregulated 15,17 , and 24 -fold, respectively in infected ticks compared to controls. The additional expression of glycine-rich proteins in the tick cement suggests a direct benefit to TBEV transmission.

\section{Proteases}

The presence of proteases in tick saliva allows the tick to actively degrade host proteins that would otherwise impede its feeding activity. Ticks primarily employ reprolysin-like metalloproteases that directly degrade fibrinogen and the extracellular matrix (Francischetti et al., 2003; Mans et al., 2003; Harnoi et al., 2007; Barnard et al., 2012; Ali et al., 2014, 2015), though they also employ dipeptidyl carboxypeptidase to inactivate bradykinin (Ribeiro and Mather, 1998; Bastiani et al., 2002), a pain-inducing peptide. Metalloproteases are required for tick feeding; when animals are vaccinated against them, ticks feed poorly or die during the feeding process, usually in the later stages (post 24-h) (Decrem et al., 2008; Ali et al., 2015).

Similar to serine protease inhibitors, upregulation of metalloproteases increases the potency of the tick saliva's anticoagulant effect. Tick-borne viruses benefit when the tick consumes greater amounts of blood faster and is able to salivate more. Although these salivary compounds are usually associated with long-term ( $24 \mathrm{~h}$ or longer) tick feeding, the presence of TBEV causes their marked downregulation in infected ticks. These included a change of $61,67,14$, and 16-fold, with the latter being the only definitive kininase detected. Of these four, those with the fold changes of 61 and 67 were still upregulated at $1 \mathrm{~h}$ of feeding, though to a lower extent (13 and 24-fold, respectively). Two genes were found downregulated (0.02 and 0.06-fold).

More infection-upregulated genes were identified at the 1-h timepoint. Of these 22 genes, fourteen had fold changes between 10 and 30, six had fold-changes between 30 and 90, and two had fold-changes of $>100$. Only three genes were found downregulated at this timepoint, with fold changes of $0.03,0.06$, and 0.05 .

In contrast, nine protease contigs were found decreased in the presence of TBEV at the $3 \mathrm{~h}$ timepoint (0.01-0.08). Five were found upregulated, with four between 10 and 15-fold and one at 19 -fold.

The expression of proteases mirrors our observations with serine protease inhibitors, where the presence of virus increases anticoagulant activity in the first two timepoints but downregulates it in the third. Likewise, this will cause the ticks to struggle to feed and force them to salivate more, thereby introducing more immunomodulatory compounds and virus as they attempt to continue feeding. They may also detach from the host without a blood meal, and may reattach to another, thus increasing virus transmission.

It should also be noted that while reprolysin-like or even astrolycin-like metalloproteases have been studied in ticks, these 
data have additionally identified several neprilysin-like proteases, a cysteine protease, and several serine proteases. The presence of serine proteases was largely upregulated at the $1 \mathrm{~h}$ timepoint and may correspond with the production of Kunitz-domain serine proteinase inhibitors. In this context the Kunitz proteins may serve to inhibit the serine proteases prior to saliva release to prevent damage to the tick salivary gland, with both assuming separate functions after release into the host.

\section{Other Tick Genes}

Several miscellaneous genes of immunological and secretory importance have also been observed in this study. Calreticulin was found to be upregulated in infected ticks during the $1 \mathrm{~h}$ timepoint. In tick saliva, calreticulin has been shown to act as an immune signaling compound in addition to its normal calciumbinding function. It was found to contribute to the transmission of Babesia (Hajdušek et al., 2013) and may be of benefit to TBEV as well.

Several antimicrobial compounds also showed changes in expression. These include defensin, which was upregulated in infected ticks at the $3 \mathrm{~h}$ timepoint (fold change of 28), as well as a $5.3 \mathrm{kDa}$ protein, which was also upregulated at $3 \mathrm{~h}$ (fold-change of 26) despite being slightly downregulated at $1 \mathrm{~h}$ feeding (foldchange 0.08). Microplusin was highly upregulated in infected ticks vs. controls (fold-change of 37), but then downregulated at $3 \mathrm{~h}$ (fold-change of 0.05 ), suggesting a decrease in tick response to the pathogen. Microplusin, however, is considered to be an antifungal and antibacterial compound (Silva et al., 2009) whereas defensin and possibly the $5.3 \mathrm{kDa}$ protein can interact with viruses. Its role, therefore, may be part of a generic tick immune response to TBEV. These antimicrobial responses are probably not meant to interact with the host during feeding, but rather the result of the salivary glands reacting to their own infection. The temporal aspects are the result of changing gene expression during feeding.

Another category of molecules that is associated with cytotoxicity is represented by antigen-5. Antigen-5 is a compound of unknown function in insect venoms that results in potent allergenic response. Here, antigen-5 was found to be upregulated at the 1-h timepoint in infected ticks and then downregulated at $3 \mathrm{~h}$ feeding, resulting in a more powerful inflammatory response, as has been observed due to TBEVinfected ticks (Thangamani et al., 2017). This may serve to drive macrophages to the site of inoculation for infection and pathogen dissemination. Contributing to this effect are several cytotoxins found to be upregulated at 1 ( 3 genes) and $3 \mathrm{~h}$ (three genes) in infected ticks. These imply increased toxicity of the saliva, and the possibility of pro-inflammatory molecules being released from damaged or necrotic cells. Upregulation of this process may serve a direct benefit to the virus through increased inflammation. However, it will be necessary to perform deeper analysis of this gene and the structure and function of its product to fully understand what role this protein has in tick feeding, and what effect its upregulation may have on the transmission of TBEV.

One additional gene of interest was a member of the 18.3 $\mathrm{kDa}$ subfamily. The role of this type of protein in tick/host interaction is not fully understood and further specificities of this protein could not be identified immediately from the sequencing data. While its function is unknown, it showed the highest upregulation of any identified gene with a fold-change of 265 at the 1-h timepoint in infected ticks vs. uninfected ticks. It was downregulated in unfed infected ticks with a fold-change of 0.02 , while its regulation was not significantly altered at the 3 h timepoint.

\section{Uncategorized Genes}

A substantial number ( $>75 \%)$ of the genes recovered could not be identified. All of those listed possess signals that identify them as secreted compounds, and they all show differential expression at one or more timepoint. Their role cannot be ascertained at present.

\section{CONCLUSION}

Tick saliva is essential to the tick's ability to modulate host hemostasis and inflammatory and immune responses against a tick during feeding and pathogen transmission. Our study clearly indicates a temporal variation in the tick salivary transcriptome expression during the first $3 \mathrm{~h}$ of feeding. Further, the pattern of expression is significantly impacted by infection of the tick with TBEV. The resulting changes to salivary transcriptome create an environment that is favorable to early viral infection and dissemination by altering compounds which affect the host immune system and hemostatic pathways. The early downregulation of lipocalins, metalloproteases and Kunitzdomain containing proteins by TBEV infection suggests that the tick will struggle to counteract host hemostatic and inflammatory responses and may be dislodged. Reattachment to another host would increase virus transmission. This strategy appears to be similar in effect to the method employed by Yersinia pestis where the bacillus impairs the ingestion of blood by infected fleas, leading them to bite multiple hosts in an attempt to feed (Hinnebusch et al., 2017). Tick saliva additionally contains compounds that facilitate tick infection, as determined by previously observed experimental evidence that tick saliva has pro-viral effect on infection; accordingly, this suggests that the virus has adapted in such a way as to not only take advantage of the immunomodulatory properties of the vector's saliva but to adjust the composition of the saliva in a way that benefits its own transmission. The exact mechanism of how the virus interacts with the vector's cellular machinery to alter transcription in this way is still unknown and it will be the subject of future investigations.

\section{DATA AVAILABILITY STATEMENT}

The raw reads used in this article were deposited at the Sequence Read Archive (SRA) of the National Center for Biotechnology Information (NCBI) under bioproject PRJNA576167, Biosample SAMN12956709, and runs SRR10240055, SRR10240054, SRR10240053, SRR10240052, SRR10240051, and SRR10240050. Seventy-three novel coding sequences have been deposited to DDBJ/EMBL/GenBank through the Transcriptome Shotgun Annotation portal under the accession GHXN00000000. 
The version described in this paper is the first version, GHXN01000000. The hyperlinked spreadsheet mapping the coding sequences, their annotation and statistical analysis can be downloaded from https://proj-bip-prod-publicread.s3. amazonaws.com/transcriptome/Ixric-TBEV-2019/Ir-tbev.zip.

\section{ETHICS STATEMENT}

The experiments involving laboratory mice were performed in accordance with the animal use protocol approved by the State Veterinary and Food Administration of the Slovak Republic (permit number 1335/12-221) and the Institute of Virology, Biomedical Research Center of the Slovak Academy of Sciences (BMC SAS).

\section{AUTHOR CONTRIBUTIONS}

ST and MK designed the experiments, provided reagents, and materials and performed the experiments. JR, $\mathrm{CH}$, and ST analyzed the data. $\mathrm{CH}$ and ST drafted the manuscript. All authors critically read and revised the manuscript.

\section{FUNDING}

This study was financially supported in part by Departmental start-up funds to ST. MK was supported by the Slovak Research

\section{REFERENCES}

Ali, A., Parizi, L. F., Guizzo, M. G., Tirloni, L., Seixas, A., Vaz, I. S. Jr., et al. (2015). Immunoprotective potential of a Rhipicephalus (Boophilus) microplus metalloprotease. Vet. Parasitol. 207, 107-114. doi: 10.1016/j.vetpar.2014.11.007

Ali, A., Triloni, L., Isezaki, M., Seixas, A., Konnai, S., Ohashi, K., et al. (2014). Reprolysin metalloproteases from Ixodes persculatus, Rhipicephalus sanguineus and Rhipicephalus microplus ticks. Exp. Appl. Acarol. 63, 559-578. doi: 10.1007/s10493-014-9796-9

Andreotti, R., Cunha, R. C., Soares, M. A., Guerrero, F. D., Leite, F. P. L., and de Leon, A. A. P. (2012). Protective immunity against tick infestation in cattle vaccinated with recombinant typsin inhibitor of Rhipicephalus microplus. Vaccine 30, 6678-6685. doi: 10.1016/j.vaccine.2012.08.066

Assumpção, T. C., Ma, D., Mizurini, D. M., Kini, R. M., Ribeiro, J. M. C., Kotsyfakis, M., et al. (2015). In vitro mode of action and anti-thrombotic activity of boophilin, a multifunctional Kunitz protease inhibitor from the midgut of a tick vector of babesiosis, Rhipicephalus microplus. PLoS Negl. Trop. Dis. 10:e0004298. doi: 10.1371/journal.pntd.0004298

Barnard, A. C., Nijhof, A. M., Gaspar, A. R. M., Neitz, A. W. H., Jongejan, F., and Maritz-Olivier, C. (2012). Expression profiling, gene silencing and transcriptional networking of metzincin metalloproteases in the cattle tick. Vet. Parasitol. 186, 403-414. doi: 10.1016/j.vetpar.2011.11.026

Bastiani, M., Hillebrand, S., Horn, F., Kist, T. B. L., Guimaraes, J. A., and Termingnoni, C. (2002). Cattle tick Boophilus microplus salivary gland contains a thiol-activated metalloendopeptidase displaying kininase activity. Insect Biochem. Mol. Biol. 32, 1439-1446. doi: 10.1016/S0965-1748(02) 00064-4

Beaufays, J., Adam, B., Decrem, Y., Prevot, P., Santinin, S., Brasseur, R., et al. (2008a). Ixodes ricinus tick lipocalins: identification, cloning, phylogenetic analysis and biochemical characterization. PLoS ONE 3:e3941. doi: 10.1371/journal.pone.0003941

Beaufays, J., Adam, B., Menten-Dedoyart, C., Fievez, L., Grosjean, A., Decrem, Y., et al. (2008b). Ir-LBP, and Ixodes ricinus tick salivary LTB4-binding and Development Agency (Contract No. APVV-18-0201) and grant VEGA 2/0047/18. JR was supported by the Intramural Research Program of the National Institute of Allergy and Infectious Diseases (Vector-Borne Diseases: Biology of Vector Host Relationship, Z01 AI000810-18).

\section{ACKNOWLEDGMENTS}

The authors wish to thank Dr. Boris Klempa and Marta Siebenstichova from the Institute of Virology, Biomedical Research Center, Slovak Academy of Sciences (SAS) for providing TBEV MBS for tick inoculations and Dr. Mirko Slovák from the Institute of Zoology SAS for tick inoculations. This work utilized the computational resources of the NIH HPC Biowulf cluster (http://hpc.nih.gov).

\section{SUPPLEMENTARY MATERIAL}

The Supplementary Material for this article can be found online at: https://www.frontiersin.org/articles/10.3389/fcimb. 2020.00041/full\#supplementary-material

Table S1 | A list of primers utilized for PCR validation for this experiment.

Figure S1 | Expression changes as determined by qPCR and the $\Delta \Delta \mathrm{Ct}$ method for ten genes showing a change in expression of $>10$ or $<0.1$-fold for at least one timepoint.

Figure S2 | Evolutionary relationships of Ixodes ricinus lipocalins.

lipocalin, interferes with host neutrophil function. PLoS ONE 3:e3987. doi: 10.1371/journal.pone.0003987

Benjamini, Y., and Hochberg, Y. (1995). Controlling the false discovery rate: a practical and powerful approach to multiple testing. J. R. Statist. Soc. B 57, 289-300. doi: 10.1111/j.2517-6161.1995.tb02031.x

Birol, I., Jackman, S. D., Nielsen, C. B., Qian, J. Q., Varhol, R., Stazyk, G., et al. (2009). De novo transcriptome assembly with ABySS. Bioinformatics 25, 2872-2877. doi: 10.1093/bioinformatics/btp367

Blisnick, A. A., Foulon, T., and Bonnet, S. I. (2017). Serine protease inhibitors in ticks: an overview of their role in tick biology and tick-borne pathogen transmission. Front. Cell Infect. Microbiol. 7:199. doi: 10.3389/fcimb.2017.00199

Bronsoms, S., Pantoja-Uceda, D., Gabrijelcic-Geiger, D., Sanglas, L., Aviles, F. X., Santoro, J., et al. (2011). Oxidative folding and structural analyses of a Kunitzrelated inhibitor and its disulfide intermediates: functional implications. J. Mol. Biol. 414, 427-441. doi: 10.1016/j.jmb.2011.10.018

Cabezas-Cruz, A., Alberdi, P., Alyllon, N., Valdes, J. J., Pierce, R., Villar, M., et al. (2016). Anaplasma phagocytophilum increases the levels of histone modifying enzymes to inhibit cell apoptosis and facilitate pathogen infection in the tick vector Ixodes scapularis. Epigenetics 11, 303-319. doi: $10.1080 / 15592294.2016 .1163460$

Chagas, A. C., Calvo, E., Rios-Velasquez, C. M., Pessoa, F. A., Medeiros, J. F., and Ribeiro, J. M. (2013). A deep insight into the sialotranscriptome of the mosquito, Psorophora albipes. BMC Genom. 14:875. doi: 10.1186/1471-2164-14-875

Chen, Z., Wang, B., Hu, J., Yang, W., Cao, Z., Zhuo, R., et al. (2013). SjAPI, the first functionally characterized Ascaris type protease inhibitor from animal venoms. PLoS ONE 8:e57529. doi: 10.1371/journal.pone.00 57529

Chen, Z. Y., Hu, Y. T., Yang, W. S., He, Y. W., Feng, J., Wang, B., et al. (2012). $\mathrm{Hg} 1$, novel peptide inhibitor specific for Kv1.3 channels from first scorpion Kunitz-type potassium channel toxin family. J. Biol. Chem. 287, 13819-13821. doi: 10.1074/jbc.M112.343996 
Chmelar, J., Kotál, J., Kopecký, J., Pedra, J. H. F., and Kotsyfakis, M. (2016). All for one and one for all on the tick-host battlefield. Trends Parasitol. 32, 368-377. doi: 10.1016/j.pt.2016.01.004

Chmelar, J., Kotál, J., Langhansová, H., and Kotsyfakis, M. (2017). Protease inhibitors in tick saliva: the role of serpins and cystatins in tick-host-pathogen interaction. Front. Cell. Infect. Microbiol. 7:2016. doi: 10.3389/fcimb.2017.00216

Dai, S. X., Zhang, A. D., and Huang, J. F. (2012). Evolution, expansion and expression of the Kunitz/BPTI gene family associated with long-term blood feeding in Ixodes scapularis. BMC Evol. Biol. 12:4. doi: 10.1186/1471-2148-12-4

Decrem, Y., Beaufays, J., Blasioli, V., Lahaye, K., Brossard, M., Vanhamme, L., et al. (2008). A family of putative metalloproteases in the salivary glands of the tick Ixodes ricinus. FEBS J. 275, 1485-1499. doi: 10.1111/j.1742-4658.2008.06308.x

Diaz-Martin, V., Manzano-Roman, R., Siles-Lucas, M., Oleaga, A., and PerezSanchez, R. (2011). Cloning, characterization and diagnostic performance of the salivary lipocalin protein TSGP1 from Ornithodoros moubata. Vet. Parasitol. 171, 163-172. doi: 10.1016/j.vetpar.2010.12.014

Drewes, C. C., Dias, R. Y. S., Hebeda, C. B., Simons, S. M., Barreto, S. A., Ferreira, J. M. Jr., et al. (2012). Actions of the Kunitz-type serine protease inhibitor Amblyomin-X on VEGF-A-induced angiogenesis. Toxicon 60, 333-340. doi: 10.1016/j.toxicon.2012.04.349

Felsenstein, J. (1985). Confidence-limits on phylogenies - an approach using the bootstrap. Evolution 39, 783-791. doi: 10.1111/j.1558-5646.1985.tb00420.x

Fialova, A., Cimburek, Z., Iezzi, G., and Kopecky, J. (2010). Ixodes ricinus tick saliva modulates tick-borne encephalitis virus infection of dendritic cells. Microbes Infect. 12, 580-585. doi: 10.1016/j.micinf.2010.03.015

Francischetti, I. M. B., Mather, R. N., and Ribeiro, J. M. C. (2003). Cloning of a salivary gland metalloprotease and characterization of gelatinase and fibrin(ogen)lytic activities in the saliva of the Lyme disease tick vector Ixodes scapularis. Biochem. Biophys. Res. Commun. 305, 869-875. doi: 10.1016/S0006-291X(03)00857-X

Francischetti, I. M. B., Sa-Nunes, A., Mans, B. J., Santos, I. M., and Ribeiro, J. M. C. (2009). The role of saliva in tick feeding. Front. Biosci. 14, 2051-2088. doi: $10.2741 / 3363$

Grabherr, M. G., Haas, B. J., Yassour, M., Levin, J. Z., Thompson, D. A., Amit, I., et al. (2011). Full-length transcriptome assembly from RNA-Seq data without a reference genome. Nat. Biotechnol. 29, 644-652. doi: 10.1038/nbt.1883

Hajdušek, O., Š́ma, R., Ayllón, N., Jalovecká, M., Perner, J., de la Fuente, J., and Kopáček, P. (2013). Interactions of the tick immune system with transmitted pathogens. Front. Cell. Infect. Microbiol. 3:26. doi: 10.3389/fcimb.2013. 00026

Harnoi, T., Sakaguchi, T., Nishikawa, Y., Xuan, X., and Fujisaki, K. (2007). Molecular characterization and comparative study of 6 salivary glands metalloproteases from the hard tick, Haemaphysalis longicornis. Comp. Biochem. Physiol. 147, 93-101. doi: 10.1016/j.cbpb.2006.12.008

Hermance, M. E., Santos, R. I., Kelly, B. C., Valbuena, G., and Thangamani, S. (2016). Immune cell targets of infection at the tick-skin interface during Powassan virus transmission. PLoS ONE 11:e0155889. doi: 10.1371/journal.pone. 0155889

Hermance, M. E., and Thangamani, S. (2015). Tick saliva enhances Powassan virus transmission to the host, influencing its dissemination and the course of disease. J. Virol. 89, 7852-7860. doi: 10.1128/JVI.01056-15

Hermance, M. E., and Thangamani, S. (2018). Tick-virus-host interactions at the cutaneous interface: the nidus of flavivirus transmission" Viruses 10:362. doi: $10.3390 / \mathrm{v} 10070362$

Hinnebusch, B. J., Jarrett, C. O., and Bland, D. M. (2017). "Fleaing' the plague: Adaptations of Yersinia pestis to its insect vector that lead to transmission. Аnnu. Rev. Microbiol. 71, 215-232. doi: 10.1146/annurev-micro-090816-093521

Hollmann, T., Kim, T. K., Tirloni, L., Radulovic, Z. M., Pinto, A. F. M., Diedrich, J. K., et al. (2018). Identification and characterization of proteins in the Amblyomma americanum tick cement cone. Int. J. Parasitol. 48, 211-224. doi: 10.1016/j.ijpara.2017.08.018

Jones, L. D., Hodgson, E., and Nuttall, P. A. (1989). Enhancement of virus transmission by tick salivary glands. J. Gen Virol. 70, 1895-1898. doi: 10.1099/0022-1317-70-7-1895

Karim, S., and Ribeiro, J. M. (2015). An insight into the sialome of the Lone Star tick, Amblyomma americanum, with a glimpse on its time dependent gene expression. PLoS One. 10:e0131292.
Karim, S., Singh, P., and Ribeiro, J. M. (2011). A deep insight into the sialotranscriptome of the gulf coast tick, Amblyomma maculatum" PLoS ONE 6:e28525. doi: 10.1371/journal.pone.0028525

Kazimírová, M., Thangamani, S., Bartíková, P., Hermance, M., Holíková, V., Štibrániová, I., et al. (2017). Tick-borne viruses and biological processes at the tick-host-virus interface. Front. Cell. Infect. Microbiol. 7:339. doi: 10.3389/fcimb.2017.00339

Kotsyfakis, M., Schwarz, A., Erhart, J., and Ribeiro, J. M. (2015). Tissue- and timedependent transcription in Ixodes ricinus salivary glands and midguts when blood feeding on the vertebrate host. Sci. Rep. 5:9103. doi: 10.1038/srep09103

Kumar, S., Stecher, G., and Tamura, K. (2016). MEGA7: molecular evolutionary genetics analysis version 7.0 for bigger datasets. Mol. Biol. Evol. 33, 1870-1874. doi: $10.1093 / \mathrm{molbev} / \mathrm{msw} 054$

Labuda, M., Austyn, J. M., Zuffova, E., Kozuch, O., Fuchsberger, N., Lysy, J., et al. (1996). Importance of localized skin infection in tick-borne encephalitis virus transmission. Virology 219, 357-366. doi: 10.1006/viro.1996.0261

Labuda, M., Jones, L. D., Williams, T., and Nuttall, P. A. (1993). Enhancement of tick-borne encephalitis virus by tick salivary gland extracts. Med. Vet. Entomol. 7, 193-196. doi: 10.1111/j.1365-2915.1993.tb00674.x

Labuda, M., Trimnell, A. R., Lickova, M., Kazimirova, M., Davies, G. M., Lissina, O., et al. (2006). An antivector vaccine protects against a lethal vector-borne pathogen. PLoS Pathog. 2:e27. doi: 10.1371/journal.ppat.0020027

Lieskovska, J., Palenikova, J., Langhansova, H., Chmelar, J., and Kopecky, J. (2018). Saliva of Ixodes ricinus enhances TBE virus replication in dendritic cells by modulation of pro-survival Akt pathway. Virology 514, 98-105. doi: 10.1016/j.virol.2017.11.008

Lieskovska, J., Palenikova, J., Širmarova, J., Elsterova, J., Kotsyfakis, M., Campos Chagas A, Calvo, E., et al. (2015). Tick salivary cystatin sialostatin L2 supresses IFN responses in mouse dendritic cells. Parasite Immunol. 37, 70-78. doi: $10.1111 /$ pim. 12162

Liu, X. Y., de la Fuente, J., Cote, M., Galindo, R. C., Moutailler, S., VayssierTaussat, M., et al. (2014). IrSPI, a tick serine protease inhibitor involved in tick feeding and Bartonella henselae infection. PLoS Negl. Trop. Dis. 8:e2993. doi: 10.1371/journal.pntd.0002993

Louw, E., van der Merwe, N., Neitz, A. W. H., and Maritz-Olivier, C. (2013). Evolution of the tissue factor pathway inhibitor-like Kunitz domain-containing protein family in Rhipicephalus microplus. Int. J. Parasitol. 43, 81-94. doi: 10.1016/j.ijpara.2012.11.006

Mans, B. J., Louw, A. I., and Neitz, A. W. H. (2003). “The major tick salivary gland proteins and toxins from the soft tick, Ornithodoros savignyi, are part of the tick lipocalin family: implications for the origins of tick toxicosis". Mol. Biol. Evol. 10, 1158-1167. doi: 10.1093/molbev/msg126

Mans, B. J., and Ribeiro, J. M. C. (2008). Function, mechanism and evolution of the moubatin-clade of soft tick lipocalins. Insect Biochem. Mol. Biol. 38, 841-852. doi: 10.1016/j.ibmb.2008.06.007

Mans, B. J., and Ribeiro, J. M. C. (2009). A novel clade of cysteinyl leukotriene scavengers in soft ticks. Insect Biochem. Mol. Biol. 38, 862-870. doi: 10.1016/j.ibmb.2008.06.002

Mourao, C. B. F., and Schwartz, E. F. (2013). Protease inhibitors from marine venomous animals and their counterparts in terrestrial venomous animals. Mar. Drugs. 11, 2069-2112. doi: 10.3390/md11062069

Neelakanta, G., Sultanah, H., Sonenshine, D. E., and Andersen, J. F. (2018) Identification and characterization of a histamine-binding lipocalin-like molecule from the relapsing fever tick Ornithodoros turicata. Insect Mol. Biol. 27, 177-187. doi: 10.1111/imb.12362

Paesen, G. C., Adams, P. L., Harlos, K., Nuttall, P. A., and Stuart, D. I. (1999). Tick histamine-binding proteins: isolation, cloning, and three-dimensional structure. Mol. Cell. 3, 661-671. doi: 10.1016/S1097-2765(00)80359-7

Perner, J., Kropáčková, S., Kopáček, P., and Ribeiro, J. M. C. (2018). Sialome diversity of ticks revealed by RNAseq of single tick salivary glands. PLoS Negl. Trop. Dis. 12:e0006410. doi: 10.1371/journal.pntd.0006410

Piesman, J. (1991). Experimental acquisition of the Lyme disease spirochete, Borrelia burgdorferi, by larval Ixodes dammini (Acari: Ixodidae) during partial blood meals. J. Med. Entomol. 28, 259-262. doi: 10.1093/jmedent/28.2.259

Preston, S. G., Majtán, J., Kouremenou, C., Rysnik, O., Burger, L. F., Cabezaz Cruz, A., et al. (2013). Novel immunomodulators from hard ticks selectively reprogramme human dendritic cell responses. PLoS Pathog. 9:e1003450. doi: 10.1371/journal.ppat.1003450 
Ribeiro, J. M., Chagas, A. C., Pham, V. M., Lounibos, L. P., and Calvo, E. (2014). An insight into the sialome of the frog-biting fly, Corethrella appendiculata. Insect. Biochem. Mol. Biol. 44, 23-32. doi: 10.1016/j.ibmb.2013.10.006

Ribeiro, J. M. C., and Mather, T. N. (1998). Ixodes scapularis: salivary kinase activity is a metallo dipeptidyl carboxypeptidase. Exp. Parasitol. 89, 213-221. doi: 10.1006/expr.1998.4296

Rodriguez-Valle, M., Moolhuijzen, P., Piper, E. K., Weiss, O., Vance, M., Bellgard, M., et al. (2013). Rhipicephalus microplus lipocalins (LRMs): genomic identification and analysis of the bovine immune response using in silico predicted $\mathrm{B}$ and $\mathrm{T}$ cell epitopes. Int. J. Parasitol. 43, 739-752. doi: 10.1016/j.ijpara.2013.04.005

Roversi, P., Johnson, S., Preston, S. G., Nunn, M. A., Paesen, G. C., Austyn, J. M., et al. (2017). Structural basis of cholesterol binding by a novel clade of dendritic cell modulators from ticks. Sci. Rep. 7:16057. doi: 10.1038/s41598-017-16413-2

Saitou, N., and Nei, M. (1987). The neighbor-joining method: a new method for reconstructing phylogenetic trees. Mol. Biol. Evol. 4, 406-425.

Sangamnatdej, S., Paesen, G. C., Slovak, M., and Nuttall, P. A. (2002). A high affinity serotonin- and histamine-binding lipocalin from tick saliva. Insect Mol. Biol. 11, 79-86. doi: 10.1046/j.0962-1075.2001.00311.x

Santibañez-Lopez, C. E., Ontano, A. Z., Harvey, M. S., and Sharma, P. P. (2018). Transcriptomic analysis of pseudoscorpion venom reveals a unique cocktail dominated by enzymes and protease inhibitors. Toxins 10:207. doi: 10.3390/toxins10050207

Schonenmeyer, A., Lucius, R., Sonnenburg, B., Brattig, N., Sabat, R., Schilling, K., et al. (2017). Modulation of human T cell responses and macrophage functions by onchosystatin, a secreted protein of the filarial nematode Ochocerca volvulus. J. Immunol. 167, 3207-3215. doi: 10.4049/jimmunol.167.6.3207

Schwarz, A., von Reumont, B. M., Erhart, J., Chagas, A. C., Ribeiro, J. M., and Kotsyfakis, M. (2013). De novo Ixodes ricinus salivary gland transcriptome analysis using two next-generation sequencing methodologies. FASEB J. 27, 4745-4756. doi: 10.1096/fj.13-232140

Silva, F. D., Rezende, C. A., Rossi, D. C. P., Esteves, E., Dyszy, F. H., Schreier, S., et al. (2009). Structure and mode of action of microplusin, a copper II-chelating antimicrobial peptide from the catle tick Ripicephalus (Boophilus) microplus. J. Biol. Chem. 284, 34735-34746. doi: 10.1074/jbc.M109.016410

Šimo, L., Kazimirova, M., Richardson, J., and Bonnet, S. I. (2017). The essential role of tick salivary glands and saliva in tick feeding and pathogen transmission. Front. Cell. Infect. Microbiol. 7:281. doi: 10.3389/fcimb.2017.00281

Slovák, M., Kazimírová, M., Siebenstichová, M., Ustaniková, K., Klempa, B., Gristun, T., et al. (2014). Survival dynamics of tick-borne encephalitis virus in Ixodes ricinus ticks. Ticks Tick Borne Dis. 4, 962-969. doi: 10.1016/j.ttbdis.2014.07.019

Smith, A. A., and Pal, U. (2014). Immunity-related genus in Ixodes scapularisperspectives from genome information. Front. Cell. Infect. Microbiol. 4:116. doi: $10.3389 /$ fcimb. 2014.00116

Soares, T. S., Oliveira, F., Torquato, R. J. S., Sasaki, S. D., Araujo, M. S., Paschoalin, T., et al. (2016). BmTI-A, a Kunitz type inhibitor from Rhipicephalus microplus able to interfere in vessel formation. Vet. Parasitol. 219, 44-52. doi: 10.1016/j.vetpar.2016.01.021

Sterba, J., Dupejova, J., Fiser, M., Vancova, M., and Grubhoffer, L. (2011). Fibrinogen-related proteins in ixodid ticks. Parasit. Vectors. 4:127. doi: 10.1186/1756-3305-4-127

Štibrániová, I., Bartíková, P., Holíková, V., and Kazimírová, M. (2019). Deciphering biological processes at the tick-host interface opens new strategies for treatment of human diseases. Front. Physiol. 10:830. doi: $10.3389 /$ fphys. 2019.00830
Team R. C. (2013). R: A Language and Environment for Statistical Computing. $R$ Foundation for Statistical Computing, Vienna, Austria.

Thangamani, S., Hermance, M. E., Santos, R. I., Slovak, M., Heinze, D., Widen, S. G., et al. (2017). Transcriptional immunoprofiling at the tickvirus-host interface during early stages of tick-borne encephalitis virus transmission. Front. Cell. Infect. Microbiol. 7:494. doi: 10.3389/fcimb.2017. 00494

Tirloni, L., Kim, T. K., Berger, M., Termignoni, C., da Silva Vaz, I. Jr., and Mulenga, A. (2019). Amblyomma americanum serpin 27 (AAS27) is a tick salivary antiinflammatory protein secreted into the host during feeding. PLoS Negl. Trop. Dis. 13:e0007660. doi: 10.1371/journal.pntd.0007660

Valdes, J. J., Cabezas-Cruz, A., Sima, R., Butterill, P. T., Ruzek, D., and Nuttall, P. A. (2016). Substrate prediction of Ixodes ricinus salivary lipocalins differentially expressed during Borrelia afzelii infection. Sci. Rep. 6:32372. doi: $10.1038 /$ srep32372

Valdes, J. J., and Moal, I. H. (2014). Prediction of Kunitz ion channel effectors and protease inhibitors from the Ixodes ricinus sialome. Ticks Tick Borne Dis. 5, 947-950. doi: 10.1016/j.ttbdis.2014.07.016

Vechtova, P., Sterbova, J., Sterba, J., Vancova, M., Rego, R. O. M., Selinger, M., et al. (2018). A bite so sweet: the glycobiology interface of tick-host-pathogen interactions. Parasit. Vectors. 11:594. doi: 10.1186/s13071-018-3062-7

Wang, Y., Li, Z., Zhou, Y., Cao, J., Zhang, H., Gong, H., et al. (2016). Specific histamine binding activity of a new lipocalin from Hyalomma asiaticum (Ixodidae) and therapeutic effects on allergic asthma in mice. Parasit. Vec. 9:506. doi: 10.1186/s13071-016-1790-0

World Health Organization (2011). Weekly Epidemiological Record (2011). No. 24, $86,241-256$.

Xu, X., Chang, B. W., Mans, B. J., Ribeiro, J. M. C., and Andersen, J. F. (2013). Structure and ligand-binding properties of the biogenic amin-binding protein from the saliva of a blood-feeding insect vector of Trypanosoma cruzi. Acta Crystallograph. D69, 105-113. doi: 10.1107/S0907444912043326

Zavadska, D., Odzelevica, Z., Karelis, G., Liepina, L., Litaurniece, Z. A., Bormane, A., et al. (2018). Tick-borne encephalitis: a 43-year summary of epidemiological and clinical data from Latvia (1973 to 2016). PLoS ONE 13:e0204844. doi: 10.1371/journal.pone.0204844

Zhang, H., Qiao, R., Gong, H., Cao, J., Zhou, Y., and Zhou, J. (2017). Identification and anticoagulant activity of a novel Kunitz-type protein HA11 from the salivary gland of the tick Hyalomma asiaticum. Exp. Appl. Acarol. 71, 71-85. doi: 10.1007/s10493-017-0106-1

Zuckerkandl, E., and Pauling, L. (1965). Molecules as documents of evolutionary history. J. Theor. Biol. 8, 357-366. doi: 10.1016/0022-5193(65)90083-4

Conflict of Interest: The authors declare that the research was conducted in the absence of any commercial or financial relationships that could be construed as a potential conflict of interest.

The reviewer JR declared a past co-authorship with one of the authors MK to the handling editor.

Copyright (c) 2020 Hart, Ribeiro, Kazimirova and Thangamani. This is an openaccess article distributed under the terms of the Creative Commons Attribution License (CC BY). The use, distribution or reproduction in other forums is permitted, provided the original author(s) and the copyright owner(s) are credited and that the original publication in this journal is cited, in accordance with accepted academic practice. No use, distribution or reproduction is permitted which does not comply with these terms. 